hep-th/0211032

AEI 2002-089

\title{
BMN Operators and Superconformal Symmetry
}

\author{
NiKLAS BeiserT \\ Max-Planck-Institut für Gravitationsphysik \\ Albert-Einstein-Institut \\ Am Mühlenberg 1, D-14476 Golm, Germany \\ nbeisert@aei.mpg.de
}

\begin{abstract}
Implications of $\mathcal{N}=4$ superconformal symmetry on Berenstein-MaldacenaNastase $(\mathrm{BMN})$ operators with two charge defects are studied both at finite charge $J$ and in the BMN limit. We find that all of these belong to a single long supermultiplet explaining a recently discovered degeneracy of anomalous dimensions on the sphere and torus. The lowest dimensional component is an operator of naive dimension $J+2$ transforming in the $[0, J, 0]$ representation of SU(4). We thus find that the BMN operators are large $J$ generalisations of the Konishi operator at $J=0$. We explicitly construct descendant operators by supersymmetry transformations and investigate their three-point functions using superconformal symmetry.
\end{abstract}




\section{Introduction and overview}

In their insightful investigation of strings on a plane-wave background [1] Berenstein, Maldacena and Nastase $(\mathrm{BMN})$ proposed a method to construct $\mathcal{N}=4 \mathrm{SU}(N)$ super Yang-Mills theory operators dual to these string states. These so-called BMN operators are single-trace operators with near extremal charge $J$ under an $\mathrm{SO}(2)$ subgroup of the $\mathrm{SO}(6) \sim \mathrm{SU}(4)$ R-symmetry. They are obtained by inserting a few impurities into the trace of an operator with extremal $\mathrm{SO}(2)$ charge. On the string theory side the extremal operator corresponds to a string in the ground state and the impurities correspond to (localised) string excitations. After a Fourier-mode decomposition of the positions of defects the field theory operators were seen to have finite anomalous dimension in the proposed limit

$$
N, J \rightarrow \infty \quad \text { with } \quad \lambda^{\prime}=\frac{g_{\mathrm{YM}}^{2} N}{J^{2}} \quad \text { and } \quad g_{2}=\frac{J^{2}}{N} \quad \text { fixed }
$$

the BMN limit. On the string theory side this corresponds to the fact that the planewave background is a Penrose limit of $\operatorname{AdS}_{5} \times S^{5}[2$. It was suggested and shown to leading order that the spectrum of scaling dimensions of these operators matches the light-cone energy spectrum of string theory.

This inspiring proposal has sparked numerous works on both the string theory and field theory side. On the string theory side the interest $[3,4,6,6,7,8,9,10$, is due to major simplifications of the calculation in the plane-wave limit. For the first time there is an opportunity to compare directly string states with field theory and test the AdS/CFT conjecture beyond supergravity. On the gauge theory side it has given rise to a number of investigations [11, 12, 13, 14, 15, 16, 17, 18, 19, 20, 21, 22, 23, 24, 25] which also brought about new insights on $\mathcal{N}=4$ SYM. Some works try to match string theory to field theory [16, 19, 26, 27, 28, 29, 30.

In 11,13, it was noticed that the BMN limit is very different from the usual 't Hooft limit in that it permits all-genus amplitudes and not just planar ones. It involves two meaningful parameters, $\lambda^{\prime}$, the coupling constant, and $g_{2}$, an effective genus counting parameter. The corrections to two-point correlation functions on the torus, $\mathcal{O}\left(g_{2}^{2}\right)$, and at one-loop, $\mathcal{O}\left(\lambda^{\prime}\right)$, were calculated in these articles. To compute the torus correction to the anomalous dimension all operators with common quantum numbers have to be redefined (mixed) in order to normalise and diagonalise their two-point functions. A crucial insight was that not only the original, single-trace BMN operators, but also similar multi-trace operators have to be taken into account [15. Including these in the diagonalisation procedure the anomalous dimension on the torus was found to be [21,22]

$$
\Delta_{n}^{J} \sim J+2+\frac{\lambda^{\prime}}{8 \pi^{2}}\left(8 \pi^{2} n^{2}+g_{2}^{2}\left(\frac{1}{6}+\frac{35}{16 \pi^{2} n^{2}}\right)\right) .
$$

In [15, 21,22] it was furthermore noticed that there are different flavours of BMN operators with two scalar impurities. With respect to the residual $\mathrm{SO}(4)$ subgroup of $\mathrm{SO}(6)$, which is orthogonal to the $\mathrm{SO}(2)$ charge subgroup, a scalar impurity transforms in the 4 irreducible representation. Two scalar impurities therefore correspond to the tensor 
product $\mathbf{4} \times \mathbf{4}=\mathbf{1}+\mathbf{3}+\overline{\mathbf{3}}+\mathbf{9}$. On general grounds one should assume that operators transforming in different irreducible representations have different properties. Nevertheless, it was observed that all 16 operators have the same anomalous dimension even on the torus. This is a clear hint at an enlarged symmetry that relates the operators. In a response to the preprints of [21,22, J. Maldacena and M. van Raamsdonk kindly pointed out that this degeneracy should be due to a symmetry that lies at the heart of $\mathcal{N}=4$ SYM, supersymmetry ${ }^{1}$. One purpose of this work is to make this statement more explicit and work out exactly how supersymmetry relates these and other operators. A first step in this direction was already taken in [20] where the vector-scalar BMN operator was related to the two-scalar BMN operators by means of supersymmetry.

Supersymmetry is not the full symmetry group of $\mathcal{N}=4$ SYM. As a massless field theory it also exhibits conformal symmetry. Due to non-renormalisation of the coupling constant in this special theory conformal symmetry is not broken by quantum corrections. Moreover, in combination with supersymmetry it enhances to superconformal symmetry. Like conformal symmetry, superconformal symmetry puts severe constraints on the correlation functions of the theory. A special role is played by the two-point and three-point functions. Two-point functions are uniquely fixed and contain information about the conformal dimension of an operator. On the string theory side the anomalous dimension corresponds to the light-cone energy. In this context superconformal symmetry has proven useful for the exact determination of the planar anomalous dimension of BMN-like operators [17]. Three-point functions, which are not fully determined, contain the structure constants for the operator product expansion (OPE). A corresponding structure for these on the string theory side, if it exists at all, is not yet known. Nevertheless, as three-point functions are well-defined observables of field theory, some of them have been explicitly computed [16, 21, 22]. Out of the six three-point functions stated in [21], two vanish and three are very similar to one another. We will explain the reason for this in terms of superconformal symmetry.

Beyond that little is known about the nature of the BMN operators. BMN have provided a heuristic method to construct them, but it is unclear how they are distinguished. It is also not known whether the operators are well-defined in terms of a $\mathcal{N}=4$ superconformal theory [14] or if the BMN limit is well-defined at all [14,21]. We will answer the first question in terms of representation theory, namely that the BMN operators with two charge defects form multiplets of $\mathcal{N}=4$ superconformal symmetry. The primary operator of the multiplet is the $\mathrm{SO}(4)$ singlet two-scalar operator discussed in [21]. It transforms under the internal $\mathrm{SO}(6)$ symmetry group as $[0, J, 0]$ and has naive scaling dimension $J+2$. All other BMN operators with two charge defects are descendants. For instance, the antisymmetric and symmetric-traceless two-scalar operators of [21] are level 2 and 4 descendants, respectively. This alternative definition of BMN operators may eventually lead to a better understanding of the BMN limit and an answer to the question whether it is a good limit of $\mathcal{N}=4 \mathrm{SYM}$.

\footnotetext{
${ }^{1}$ This was also noted in [1] and a revised version of 22 .
} 
For the primary operators we find the explicit form

$$
\mathcal{O}_{n}^{J}=\frac{N_{0}^{-J-2}}{2 \sqrt{J+3}} \sum_{p=0}^{J} \sum_{m=1}^{6} \cos \frac{\pi n(2 p+3)}{J+3} \operatorname{Tr} \Phi_{m} Z^{p} \Phi_{m} Z^{J-p}, \quad 0<n<\frac{J+3}{2},
$$

at one-loop and at the planar level. The phase factors are determined such that the operators are fully orthonormal at this order even at finite charge $J$. From this expression we derive the form of all bosonic operators by supersymmetry.

Having resolved the form of the exact phase factors at finite $J$ it is natural to investigate these generalised operators at finite $J$. They form a sector of operators being nearly protected. Its large charge limit describes strings in the plane-wave background. In the low charge regime we will discover the Konishi operator $(J=0, n=1)$ and a couple of dimension four operators which have been investigated in the recent years. We also find an expression for the one-loop planar scaling dimension of the operators which interpolates smoothly between the two regimes

$$
\Delta_{n}^{J}=J+2+\frac{g_{\mathrm{YM}}^{2} N}{8 \pi^{2}} 8 \sin ^{2} \frac{\pi n}{J+3} .
$$

For $J=0, n=1$ it reproduces precisely the Konishi anomalous dimension $3 g_{\mathrm{YM}}^{2} N / 4 \pi^{2}$ and in the BMN limit it approaches $J+2+\lambda^{\prime} n^{2}$.

This paper is organised as follows. In Sec. 2 we will use counting arguments to demonstrate how BMN operators fill out multiplets of $\mathcal{N}=4 \mathrm{SYM}$. This will lead to an alternative and more general definition of BMN operators. In Sec. 3 we determine the form of these operators in terms of fields. It will be seen that the operators are meaningful objects even at finite charge. We find an expression for their anomalous dimensions which, in particular, interpolates between the BMN limit and the Konishi operator. In Sec. 4 we will work out the expressions for the descendant operators by supersymmetry transformations. This makes the observed degeneracy of anomalous dimensions manifest and is shown to yield non-trivial relations among their mixing matrices. Superconformally covariant correlation functions involving the BMN operators are considered in Sec. 5. We will show how this leads to relations between three-point functions. In Sec. 6] we discuss the results of the preceding sections and draw conclusions. This leads us to a classification of operators in $\mathcal{N}=4 \mathrm{SYM}$ inspired by, but not limited to the BMN limit.

\section{$2 \quad$ BMN Multiplets}

Berenstein, Maldacena and Nastase have introduced the operators [1]

$$
\sum_{p=1}^{J} e^{2 \pi i n p / J} \operatorname{Tr} \phi_{i} Z^{p} \phi_{j} Z^{J-p}
$$

of $\mathcal{N}=4 \mathrm{SYM}$ and suggested that they behave nicely in the limit (1.1). They have since been called 'BMN operators' ${ }^{2}$. They belong to the class of operators with two defects.

\footnotetext{
${ }^{2}$ Operators with more than two insertions in the string of $Z$ s have also been proposed. We do not consider these throughout the work and will always refer to operators with two impurities.
} 
In this section we will work out the multiplet structure of such single-trace operators with no more than two charge defects. This will clarify the nature of these operators in terms of representation theory of $\mathcal{N}=4 \mathrm{SYM}$.

A charge defect is explained as follows. The internal symmetry group $\mathrm{SO}(6)$ is split into $\mathrm{SO}(2) \times \mathrm{SO}(4)$. The defect charge is the difference between the naive scaling dimension of an operator and its $\mathrm{SO}(2)$ charge. There are six scalars in $\mathcal{N}=4$ SYM transforming in the vector representation of $\mathrm{SO}(6)$. They will be denoted by $Z=\frac{1}{\sqrt{2}}\left(\Phi_{5}+i \Phi_{6}\right)$, $\phi_{1,2,3,4}=\Phi_{1,2,3,4}$ and $\bar{Z}=\frac{1}{\sqrt{2}}\left(\Phi_{5}-i \Phi_{6}\right)$. They carry charge $+, 0,-$ and transform under $\mathrm{SO}(4)$ as $\mathbf{1}, \mathbf{4}, \mathbf{1}$, respectively. Therefore, the only scalar without a charge defect is $Z$.

To enumerate the operators, it is convenient to use Young tableau notation of $\mathrm{SO}(6)$ representations instead of the more common Dynkin labels notation $[a, b, c]$. An $\mathrm{SO}(6)$ Young tableau consists of three horizontal lines of boxes with decreasing size. We will denote such a Young tableau by $(a, b, c)$ with $a \geq b \geq|c|^{3}$. It corresponds to the Dynkin labels $[b+c, a-b, b-c]$. Young tableaux are useful in this context because the maximum $\mathrm{SO}(2)$ charge in a representation can be read off directly as the number of boxes in the first row, $a$. Furthermore, the weights with maximum charge form a representation under the transverse $\mathrm{SO}(4)$ given by the lower two rows, $(b, c)$.

First we consider single-trace operators made up of $\Delta$ scalars with charge $J \geq \Delta-2$, i.e. no more than two charge defects. The scalars transform under the fundamental representation $(1,0,0)$ of $\mathrm{SO}(6)$, a single box. The product of $\Delta$ scalars is thus a sum of representations with Young tableaux of $\Delta, \Delta-2, \Delta-4, \ldots$ boxes. As we are interested only in operators with at most two charge defects, $J \geq \Delta-2$, and the charge in a representation is bounded, $J \leq a$, there are only very few representations to be considered. We present them in the following table.

\begin{tabular}{lc|ccc} 
Young tab. & Dynkin l. & $\Delta$ & $\Delta-1$ & $\Delta-2$ \\
\hline$(\Delta, 0,0)$ & {$[0, \Delta, 0]$} & $\mathbf{1}$ & $\mathbf{4}$ & $\mathbf{1 + 9}$ \\
$(\Delta-1,1,0)$ & {$[1, \Delta-2,1]$} & & $\mathbf{4}$ & $\mathbf{4} \times \mathbf{4}$ \\
$(\Delta-2,0,0)$ & {$[0, \Delta-2,0]$} & & & $\mathbf{1}$ \\
$(\Delta-2,1,+1)$ & {$[2, \Delta-3,0]$} & & & $\mathbf{3}$ \\
$(\Delta-2,1,-1)$ & {$[0, \Delta-3,2]$} & & & $\overline{\mathbf{3}}$ \\
$(\Delta-2,2,0)$ & {$[2, \Delta-4,2]$} & & & $\mathbf{9}$
\end{tabular}

In the three columns labelled $\Delta-n$ we list all constituent weights of charge $\Delta-n$ and the representations of $\mathrm{SO}(4)$ they form. Next, we count the number of operators made from $\Delta$ scalars in a single trace with charge $J \geq \Delta-2$. We note that most of the scalars must be $Z \mathrm{~s}$, the only field without a defect-charge. The scalars $\phi_{i}$ carry one charge defect and there can only be two such impurities, $\phi_{i}, \phi_{j}$. All combinations of indices $i, j$ and positions of defects must be considered taking into account the cyclicity of the trace. The indices take 4 values and a tensor product of two can be decomposed into the singlet $(\mathbf{1})$, antisymmetric $(\mathbf{3}+\overline{\mathbf{3}})$ and symmetric-traceless $(\mathbf{9})$ part. In the trace only the distance between the two impurities matters. The upper bound for the distance $[(\Delta-2) / 2]$ or $[(\Delta-3) / 2]$ for symmetric or antisymmetric combinations of the impurities.

\footnotetext{
${ }^{3}$ Representations with $c>0$ are chiral, they have anti-chiral partners with $c<0$.
} 
We obtain the following table where we list the number of $\mathrm{SO}(4)$ multiplets at charge $J=\Delta, \Delta-1, \Delta-2^{4}$.

\begin{tabular}{c|rrr}
$\mathrm{SO}(4)$ & $\Delta$ & $\Delta-1$ & \multicolumn{1}{c}{$\Delta-2$} \\
\hline $\mathbf{1}$ & 1 & & $1+[\Delta / 2]$ \\
$\mathbf{4}$ & & 1 & \\
$\mathbf{3}$ & & & {$[(\Delta-1) / 2]$} \\
$\overline{\mathbf{3}}$ & & & {$[(\Delta-1) / 2]$} \\
$\mathbf{9}$ & & & $1+[(\Delta-2) / 2]$
\end{tabular}

By comparing both tables we see that the single operator at charge $J=\Delta$ must transform in the $[0, \Delta, 0]$ representation. It must be accompanied by a complete multiplet of $\mathrm{SO}(6)$. From (2.2) we conclude that also the $\mathbf{4}$ operators at charge $J=\Delta-1$ and $\mathbf{1}+\mathbf{9}$ operators at charge $J=\Delta-2$ belong to the same multiplet. These are the operators $\operatorname{Tr} Z^{J}, \operatorname{Tr} \phi_{i} Z^{J-1}, \ldots$, we will come back the explicit form in the next section. The primary operator at charge $J=\Delta$ corresponds to a string-vacuum, we will thus call its multiplet the 'vacuum multiplet'. The $\mathbf{4}$ operators at charge $J=\Delta-1$ and the $\mathbf{1}+\mathbf{9}$ operators at charge $J=\Delta-2$ correspond to strings with one or two excited zero-modes. Despite that, we will collectively refer to them as vacuum operators because they belong to one and the same multiplet. It is a general feature of the considered multiplets that they contain not only a fixed number of excited non-zero modes (none in this case), but also an arbitrary amount of excited zero-modes.

All operators at charge $J=\Delta-1$ belong to the multiplet $[0, \Delta, 0]$, hence the multiplet $[1, \Delta-2,1]$ is not realised ${ }^{5}$. Further operators not contained in the vacuum multiplet appear at charge $J=\Delta-2$ and we can identify their corresponding $\mathrm{SO}(6)$ representations. The following table summarises the multiplets and their multiplicities up to two charge defects.

\begin{tabular}{c|cc|l} 
Dynkin 1. & $\mathrm{SO}(4)$ & $J$ & multiplicity \\
\hline$[0, \Delta, 0]$ & $\mathbf{1}$ & $\Delta$ & 1 \\
{$[0, \Delta-2,0]$} & $\mathbf{1}$ & $\Delta-2$ & {$[\Delta / 2]$} \\
{$[2, \Delta-3,0]$} & $\mathbf{3}$ & $\Delta-2$ & {$[(\Delta-1) / 2]$} \\
{$[0, \Delta-3,2]$} & $\overline{\mathbf{3}}$ & $\Delta-2$ & {$[(\Delta-1) / 2]$} \\
{$[2, \Delta-4,2]$} & $\mathbf{9}$ & $\Delta-2$ & {$[(\Delta-2) / 2]$}
\end{tabular}

A central question of this paper is to what multiplets of supersymmetry the BMN operators belong. Due to the multiplicities one can at this point conjecture that the antisymmetric $(\mathbf{3}+\overline{\mathbf{3}})$ and symmetric-traceless $(\mathbf{9})$ operators are level 2 and 4 supersymmetry descendants of the singlet operators $(\mathbf{1})$. The reason is that two supersymmetry variations raise the dimension $\Delta$ by one and the multiplicities of the shifted multiplets match.

Due to supersymmetry it is not enough to consider only the operators made from scalars, but spinors and gauge fields (in covariant derivatives and field strengths) have to be taken into account as well. The 16 spinors have dimension $\frac{3}{2}$, half of which $(\psi)$ have charge $+\frac{1}{2}$ and half of which $(\bar{\psi})$ have charge $-\frac{1}{2}$. Therefore, $\psi$ carries one charge defect while $\bar{\psi}$ carries two. The 4 derivatives $\mathcal{D}_{\mu}$ carry one charge defect and the field

\footnotetext{
${ }^{4}$ There is one additional $\mathrm{SO}(4)$ singlet due to the insertion of one double-defect impurity $\bar{Z}$.

${ }^{5}$ The symmetry of $[1, \Delta-2,1]$ seems to be incompatible with the cyclicity of a single trace.
} 
strength $F_{\mu \nu}$ carries two. The following table states the number of all bosonic single-trace operators with two charge defects ${ }^{6}$.

\begin{tabular}{|c|c|c|c|c|}
\hline level & impurities & $\mathrm{SO}(3,1)$ & $\mathrm{SO}(4)$ & multiplicity \\
\hline 0 & $\phi \phi, \bar{Z}$ & 1 & 1 & $1+[\Delta / 2]$ \\
\hline 2 & $\begin{array}{c}\psi \psi, F \\
\phi \phi\end{array}$ & $\begin{array}{c}3+\overline{3} \\
1\end{array}$ & $\begin{array}{c}1 \\
3+\overline{3}\end{array}$ & $\begin{aligned} 1+ & {[(\Delta-1) / 2] } \\
& {[(\Delta-1) / 2] }\end{aligned}$ \\
\hline 4 & $\begin{array}{l}\phi \phi \\
\psi \psi \\
\psi \psi \\
\mathcal{D D} \mathcal{D} \\
\end{array}$ & $\begin{array}{ll}1 & \\
1 & \\
& 35 \\
9 & \\
\end{array}$ & $\begin{array}{l}9 \\
1 \\
1 \\
\end{array}$ & $\begin{aligned} 1+ & {[(\Delta-2) / 2] } \\
& {[(\Delta-2) / 2] } \\
& {[(\Delta-2) / 2] } \\
1+ & {[(\Delta-2) / 2] }\end{aligned}$ \\
\hline 6 & $\begin{array}{l}\mathcal{D D} \\
\psi \psi\end{array}$ & $\begin{array}{c}3+\overline{3} \\
1\end{array}$ & $\begin{array}{c}1 \\
3+\overline{3}\end{array}$ & $\begin{array}{r} \\
{[(\Delta-3) / 2]} \\
1+[(\Delta-3) / 2]\end{array}$ \\
\hline 8 & $\mathcal{D D}$ & 1 & 1 & $1+[(\Delta-4) / 2]$ \\
\hline $2,4,6$ & $\begin{array}{l}\mathcal{D} \phi \\
\psi \psi\end{array}$ & $\begin{array}{l}4 \\
4\end{array}$ & $\begin{array}{l}4 \\
4\end{array}$ & $\begin{array}{l}1+[(\Delta-1) / 2]+[(\Delta-2) / 2] \\
1+[(\Delta-3) / 2]\end{array}$ \\
\hline
\end{tabular}

From this table we need to subtract components of the vacuum multiplet, these are indicated as a ' $1+$ ' in the table. They are $\mathrm{SO}(6)(\phi \phi)$, supersymmetry $(\psi \psi)$, derivative $(\mathcal{D D})$ or mixed $(\mathcal{D} \phi)$ descendants of the vacuum operators. Apart from these we see that the remaining modes fit nicely into supermultiplets generated by 8 fermionic generators. This is a strong indication that all these operators belong to $[\Delta / 2]$ supermultiplets of $\mathcal{N}=4$ SYM whose primary operators, $\mathcal{O}_{n}^{\Delta-2}$, transform in the $[0, \Delta-2,0]$ representation of $\mathrm{SO}(6)$ and have naive dimension $\Delta$. We will show this explicitly in Sec. 4, for the time being we take it as a fact. These $[\Delta / 2]$ supermultiplets contain all BMN operators with two defects, consequently they will be called 'BMN multiplets'. This argument can also be turned around to define BMN operators:

The multiplets of BMN operators are the supermultiplets of single-trace operators whose primary operators have dimension $\Delta$ and transform in the $[0, \Delta-2,0]$ representation of $\mathrm{SO}(6)$. There are $[\Delta / 2]$ such multiplets.

In fact, this is a generalisation of BMN operators which previously were defined only in the BMN limit. This definition is universal. Furthermore it describes only operators which are similar to the ones proposed by BMN ${ }^{7}$, this will be seen in the next section. In terms of representation theory of $\mathrm{SU}(2,2 \mid 4)$ this multiplet belongs to the A series of unitary irreducible representations [31,32. It is at the unitarity bound, therefore the anomalous dimension is strictly non-negative. Furthermore, the long supermultiplet of $2^{16} \times \operatorname{dim}[0, \Delta-2,0]$ operators decomposes into a sum of shorter multiplets at vanishing coupling constant $g_{\mathrm{YM}}=0$ 33. In contrast, the vacuum multiplet is a short supermultiplet of the $\mathrm{C}$ series. As such it is protected, its scaling dimension cannot be modified by quantum corrections.

\footnotetext{
${ }^{6}$ At level 8 the operator with two derivatives at the same place has been omitted because it can be written as a linear combination of the other operators via the equations of motion. The $\mathbf{3 5}$ at level 4 is a self-dual four-form of $\mathrm{SO}(7,1)$ which has not been decomposed to $\mathrm{SO}(3,1) \times \mathrm{SO}(4)$ irreps.

${ }^{7}$ It also includes descendant operators with more than two impurities, these are operators with additional zero-modes, but only two non-zero modes $(+n,-n)$
} 


\section{$3 \quad$ BMN Operators at finite charge}

In the last section we have found that the single-trace operators with no more than two defects form one short supermultiplet $[0, \Delta, 0]$ and $[\Delta / 2]$ long supermultiplets $[0, \Delta-2,0]$. We need to transform this abstract finding into an explicit form for the operators.

The unique operator without a charge defect is $\operatorname{Tr} Z^{\Delta}$. It is the primary operator of the supermultiplet $[0, \Delta, 0]$ and its $\mathrm{SO}(6)$ descendants with one and two defects are ${ }^{8}$

$$
\begin{aligned}
\mathcal{Q}^{J} & =\frac{N_{0}^{-J}}{\sqrt{J}} \operatorname{Tr} Z^{J} \\
\mathcal{Q}_{i}^{J,[1]} & =N_{0}^{-J} \operatorname{Tr} \phi_{i} Z^{J-1} \\
\mathcal{Q}^{J,[2]} & =\frac{N_{0}^{-J}}{2 \sqrt{2} \sqrt{J+1}}\left(\sum_{p=0}^{J-2} \operatorname{Tr} \phi_{i} Z^{p} \phi_{i} Z^{J-2-p}-4 \operatorname{Tr} \bar{Z} Z^{J-1}\right) \\
\mathcal{Q}_{(i j)}^{J,[2]} & =\frac{N_{0}^{-J}}{\sqrt{2} \sqrt{J-1}} \sum_{p=0}^{J-2} \operatorname{Tr} \phi_{(i} Z^{p} \phi_{j} Z^{J-2-p}
\end{aligned}
$$

with a normalisation constant

$$
N_{0}=\sqrt{\frac{g_{\mathrm{YM}}^{2} N}{8 \pi^{2}}} .
$$

From the discussion of the last section we know that there are $[\Delta / 2] \mathrm{SO}(4)$ singlet operators with two scalar impurities but we do not know their explicit form. All of these operators have common quantum numbers and mix with each other. The explicit form of the operators can be determined by requiring two things. First, the two-point function of such operators should be canonically normalised at tree level and, second, the operators should have definite scaling dimension. From a one-loop, planar calculation at finite $J$ we find the forms of the BMN operators with two scalar impurities ${ }^{9} 10$

$$
\begin{aligned}
\mathcal{O}_{n}^{J} & =\frac{N_{0}^{-J-2}}{\sqrt{J+3}}\left[\frac{1}{2} \sum_{p=0}^{J} \cos \frac{\pi n(2 p+3)}{J+3} \operatorname{Tr} \phi_{i} Z^{p} \phi_{i} Z^{J-p}-2 \cos \frac{\pi n}{J+3} \operatorname{Tr} \bar{Z} Z^{J+1}\right], \\
\mathcal{O}_{[i j], n}^{J-1,(1)} & =\frac{N_{0}^{-J-2}}{\sqrt{J+2}} \sum_{p=0}^{J} i \sin \frac{\pi n(2 p+2)}{J+2} \operatorname{Tr} \phi_{[i} Z^{p} \phi_{j]} Z^{J-p} \\
\mathcal{O}_{(i j), n}^{J-2,(2)} & =\frac{N_{0}^{-J-2}}{\sqrt{J+1}} \sum_{p=0}^{J} \cos \frac{\pi n(2 p+1)}{J+1} \operatorname{Tr} \phi_{(i} Z^{p} \phi_{j)} Z^{J-p} .
\end{aligned}
$$

${ }^{8}$ The superscript $J$ denotes the $\mathrm{SO}(2)$ charge of the primary operator of the supermultiplet. A number in square brackets denotes an $\mathrm{SO}(6)$ descendant and a number in round brackets denotes a supersymmetry descendant. The subscripts are mode numbers as well as $\mathrm{SO}(3,1)$ spacetime and $\mathrm{SO}(4)$ internal indices. Round brackets correspond to symmetric-traceless combinations and square brackets to antisymmetric combinations.

${ }^{9}$ We find $J+3$ in the denominator of the cosine of the singlet operator. This is (hopefully) the upper bound in the sequence of previously suggested denominators $J[1], J+1[15$ and $J+2[19]$.

${ }^{10}$ These operators are made out of scalars only. In fact, one should also consider spinor and vector operators belonging to the same $S O(4)$ representations. We expect that mixing between these operators becomes relevant at higher loops, it is irrelevant in the present investigation. 
They correspond to the operators $\mathcal{O}_{1, n}^{J}, \mathcal{O}_{[i j], n}^{J}$ and $\mathcal{O}_{(i j), n}^{J}$ of [21], the modified notation is more suitable for the multiplet structure of the operators. Interestingly, the one-loop computation at finite $J$ requires different phase factors for the three flavours of operators to acquire complete diagonalisation. This is not an issue in the BMN limit, where the precise form of the phase factors becomes irrelevant. We note that our phase factors do not agree with the phase factors used in the $1 / J$ expansion of [19. This is not a contradiction, however, as the operators in [19] are explicitly not diagonalised (bare), whereas our claim is that the operators (3.3) are the one-loop planar approximation to the full operators. We will come back to this issue in the conclusions.

The mode numbers $n$ obey the symmetries (at this point we shift the dimension of the antisymmetric and symmetric-traceless operators by one and two, respectively)

$$
\begin{array}{ll}
\mathcal{O}_{n}^{J}=+\mathcal{O}_{-n}^{J}=-\mathcal{O}_{J+3+n}^{J}, & \mathcal{O}_{(J+3) / 2}^{J}=0, \\
\mathcal{O}_{[i j], n}^{J,(1)}=-\mathcal{O}_{[i j],-n}^{J,(1)}=-\mathcal{O}_{[i j], J+3+n}^{J,(1)}, & \mathcal{O}_{[i j],(J+3) / 2}^{J,(1)}=0, \\
\mathcal{O}_{(i j), n}^{J,(2)}=+\mathcal{O}_{(i j),-n}^{J,(2)}=-\mathcal{O}_{(i j), J+3+n}^{J,(2)}, & \mathcal{O}_{(i j),(J+3) / 2}^{J,(2)}=0
\end{array}
$$

and the zero-modes vanish or belong to the vacuum multiplet

$$
\mathcal{O}_{0}^{J}=\sqrt{2} \mathcal{Q}^{J+2,[2]}, \quad \mathcal{O}_{[i j], 0}^{J,(1)}=0, \quad \mathcal{O}_{(i j), 0}^{J,(2)}=\sqrt{2} \mathcal{Q}_{(i j)}^{J+4,[2]} .
$$

To avoid complications concerning linearly dependent operators we constrain the mode number of the operator to the first Brillouin zone $0<n<(J+3) / 2$. The number of modes, $[(J+2) / 2]$, matches the number of different operators as discussed in the last section. Hence the vacuum and BMN operators form a complete basis for the space of all operators with two scalar impurities. It is interesting to see that all operators participate in one and the same mode decomposition. There is no exceptional operator at two defects, the operator $\operatorname{Tr} \bar{Z} Z^{J+1}$ mixes with the other operators. This means that in the BMN limit essentially all operators behave similarly, e.g. have finite anomalous dimensions.

There is a nice alternative way to write the singlet BMN operator

$$
\mathcal{O}_{n}^{J}=\frac{N_{0}^{-J-2}}{2 \sqrt{J+3}} \sum_{p=0}^{J} \sum_{m=1}^{6} \cos \frac{\pi n(2 p+3)}{J+3} \operatorname{Tr} \Phi_{m} Z^{p} \Phi_{m} Z^{J-p}
$$

involving a sum over all six scalar fields $\Phi_{m}$. Intriguingly, it fails for the zero-mode, $n=0$, which belongs to a different multiplet. We have calculated the two-point functions of these operators up to one loop and on the sphere using the effective vertices of [21] and found

$$
\begin{aligned}
\left\langle\overline{\mathcal{O}}_{m}^{J}(x) \mathcal{O}_{n}^{K}(y)\right\rangle & =\frac{\delta_{J K} \delta_{m n}}{(x-y)^{2 \Delta_{n}^{J}}}, \\
\left\langle\overline{\mathcal{O}}_{[i j], m}^{J,(1)}(x) \mathcal{O}_{[k l], n}^{K,(1)}(y)\right\rangle & =\frac{\delta_{J K} \delta_{m n} \delta_{i[k} \delta_{l] j}}{(x-y)^{2 \Delta_{n}^{J}+2}} \\
\left\langle\overline{\mathcal{O}}_{(i j), m}^{J,(2)}(x) \mathcal{O}_{(k l), n}^{K,(2)}(y)\right\rangle & =\frac{\delta_{J K} \delta_{m n} \delta_{i(k} \delta_{l) j}}{(x-y)^{2 \Delta_{n}^{J}+4}}
\end{aligned}
$$


The planar one-loop scaling dimension is given by

$$
\Delta_{n}^{J}=J+2+\frac{g_{\mathrm{YM}}^{2} N}{8 \pi^{2}} 8 \sin ^{2} \frac{\pi n}{J+3} .
$$

Interestingly, we notice that the anomalous piece is strictly bounded from above by $g_{\mathrm{YM}}^{2} N / \pi^{2}$ (at this order in perturbation theory).

We see that the singlet, $\mathcal{O}_{n}^{J}$, antisymmetric, $\mathcal{O}_{[i j], n}^{J,(1)}$, and symmetric-traceless, $\mathcal{O}_{(i j), n}^{J,(2)}$, operators of naive dimension $J+2, J+3$ and $J+4$ have the same anomalous dimension $\delta \Delta_{n}^{J}$. This supports our previous conjecture that these operators belong to the same supermultiplet which will be shown explicitly by supersymmetry variations in the next section ${ }^{11}$. This will prove to all orders (in $g_{\mathrm{YM}}, 1 / J$ and $1 / N$ ) the equality of anomalous dimensions of the operators ${ }^{12}$. We will also demonstrate that the redefinitions of operators in $1 / N$ [21, 22], see footnote 11, obey certain relations which guarantee that the redefined operators are superpartners even at $\mathcal{O}\left(1 / N^{2}\right)$.

In the context of the BMN limit one usually considers operators of a common naive dimension $J+2$. At finite charge the singlet, antisymmetric and symmetric-traceless operators have non-degenerate anomalous dimensions $\delta \Delta_{n}^{J}, \delta \Delta_{n}^{J-1}$ and $\delta \Delta_{n}^{J-2}$. If the BMN limit of $\delta \Delta_{n}^{J}$ exists to all orders in perturbation theory, $J$ can appear only in the combinations $\lambda^{\prime}$ and $g_{2}$. Substituting $J \rightarrow J-1, J-2$ only gives rise to $\mathcal{O}(1 / J)$ corrections which are irrelevant in the strict BMN limit. Therefore all flavours of BMN operators have degenerate anomalous dimensions in the BMN limit. From (3.7) and (3.8) we see this explicitly, namely the anomalous dimension of all operators is $\lambda^{\prime} n^{2}$.

Taking a closer look at operators with small $J$ we observe that the $J=0$ BMN supermultiplet including the operators $\left(\mathcal{O}_{1}^{0}, \mathcal{O}_{[i j], 1}^{0,(1)}, \mathcal{O}_{(i j), 1}^{0,(2)}\right)$ is the Konishi multiplet (see e.g. [34,35]). From (3.8) we obtain the correct anomalous dimension

$$
\delta \Delta_{1}^{0}=\frac{3 g_{\mathrm{YM}}^{2} N}{4 \pi^{2}}
$$

The dimension 3 operator has apparently been left out in the literature, we find the anomalous dimension $\delta \Delta_{1}^{1}=g_{\mathrm{YM}}^{2} N / 2 \pi^{2}$. The two $J=2$ BMN operators coincide with the operators that have been studied in [15], we obtain the same planar anomalous dimensions

$$
\delta \Delta_{1,2}^{2}=\frac{g_{\mathrm{YM}}^{2} N(5 \mp \sqrt{5})}{8 \pi^{2}}
$$

This is a new connection between the BMN limit and operators of a small dimension. As we shall see later, the BMN operators with large charge $J$ behave much like big brothers of the Konishi operator in other respects as well. Here, it has proven useful to consider a whole class of operators with a fixed number of defects. For instance, we have found an

\footnotetext{
${ }^{11}$ In fact, these operators are just the leading order (in $g_{\mathrm{YM}}$ and $1 / N$ ) approximations to the exact operators which are exactly related by supersymmetry. We will show that the approximate operators, as given in (3.3), are related by supersymmetry. As being related by supersymmetry is a discrete statement, it cannot be changed in perturbation theory. In other words, although the operators receive corrections, they will always belong to the same supermultiplet.

${ }^{12}$ Due to the $\mathrm{SU}(2,2 \mid 4)$ commutator $[D, Q]=\frac{1}{2} Q$ the scaling dimensions of all members of a supermultiplet differ by half integers. Consequently, their anomalous pieces are exactly degenerate.
} 
expression for a large class of operators and their anomalous dimension that interpolates between two regimes. The number of defects could turn out to be a useful classification for operators of $\mathcal{N}=4 \mathrm{SYM}$ in general. We will address this issue in Sec. 6 .

\section{Supersymmetry}

To show explicitly how the BMN operators are related we will determine the bosonic supersymmetry descendants of the singlet BMN operator. It is demonstrated that all flavours of two-impurity BMN operators addressed in Sec. 2] belong to a single long supermultiplet of $\mathcal{N}=4 \mathrm{SYM}$. This puts the previously discovered degeneracy of anomalous dimension on firm ground.

We will start by short review of superspace gauge theory. This will be required as we would like to take supersymmetry descendants by acting with fermionic derivatives. As opposed to (global) supersymmetry it enables us to perform variations on a single operator in a correlator and not all operators together. Once a correlation function with full dependence on the fermionic coordinates is known it enables us to derive correlators for all descendant operators. The form of some correlation functions will be inferred from superconformal symmetry in Sec. 5 ,

\subsection{Review of Superspace Gauge Theory in $D=9+1$}

We start by reviewing $\mathcal{N}=1$ gauge theory in $D=9+1$ superspace, cf. [36, 37, 38, 39. These papers state that the constraints that have to be imposed on this theory force the gauge fields on shell. Therefore, the constraints cannot be solved and the theory is not suited well for quantisation. We are not trying to accomplish this here, all computations of correlation functions were done in non-supersymmetric component language as in [11,21]. Nevertheless, it is a nice framework which allows one to write down operators and all their supersymmetry descendants in a compact way. We prefer a ten-dimensional notation over a four-dimensional one, essentially because it does not have a distinction of chiralities, resulting in more unified expressions. In App. A we present our notation and a collection of useful identities.

Superspace is parametrised by the $\mathbf{1 0}$ real bosonic coordinates $X^{M}$ and the $\mathbf{1 6}$ real fermionic coordinates $\Theta^{A}$. Translations on this space are generated by the operators

$$
i P_{M}=\partial_{M}, \quad i Q_{A}=\partial_{A}-\Sigma_{A B}^{M} \Theta^{B} \partial_{M} .
$$

The corresponding supertranslation covariant derivatives are

$$
D_{M}=\partial_{M}, \quad D_{A}=\partial_{A}+\Sigma_{A B}^{M} \Theta^{B} \partial_{M}
$$

The fermionic derivatives satisfy the anticommutation relation

$$
\left\{D_{A}, D_{B}\right\}=2 \Sigma_{A B}^{M} D_{M},
$$

while commutators with a bosonic derivative $D_{M}$ vanish. 
On this space we define a gauge theory with the supercovariant derivatives

$$
\mathcal{D}_{M}=D_{M}+i A_{M}, \quad \mathcal{D}_{A}=D_{A}+i A_{A} .
$$

Under a gauge transformation $g(X, \Theta)$ the gauge fields transform canonically according to $A \mapsto g A g^{-1}+i D g g^{-1}$. The covariant field strengths of the gauge field are

$$
\begin{aligned}
\left\{\mathcal{D}_{A}, \mathcal{D}_{B}\right\} & =2 \Sigma_{A B}^{M} \mathcal{D}_{M}+i F_{A B}, \\
{\left[\mathcal{D}_{A}, \mathcal{D}_{M}\right] } & =i F_{A M} \\
{\left[\mathcal{D}_{M}, \mathcal{D}_{N}\right] } & =i F_{M N}
\end{aligned}
$$

We can now impose a constraint on the gauge field, namely that the field strength $F_{A B}$ vanishes

$$
F_{A B}=0 .
$$

This field strength can be decomposed into two $\mathrm{SO}(9,1)$ irreps, $\mathbf{1 0}$ and $\mathbf{1 2 6}$. The vanishing of the $\mathbf{1 0}$ part determines the bosonic gauge field $A_{M}$ in terms of the fermionic one. The 126 part has much more drastic consequences as it not only reduces the number of independent components, but also implies equations of motion for the gauge field. Before stating these, we present two important consequences of the constraint

$$
\begin{aligned}
& {\left[\mathcal{D}_{A}, \mathcal{D}_{M}\right]=i F_{A M}=i \Sigma_{M, A B} \Psi^{B}} \\
& \left\{\mathcal{D}_{A}, \Psi^{B}\right\}=\frac{i}{2} \tilde{\Sigma}_{A}^{M N, B}\left[\mathcal{D}_{M}, \mathcal{D}_{N}\right]=-\frac{1}{2} \tilde{\Sigma}^{M N, B}{ }_{A} F_{M N} .
\end{aligned}
$$

The first shows that the $\mathbf{1 4 4}$ part of the field strength $F_{A M}$ is zero, it can be proved by using the Jacobi identity and inserting the constraint. The second one can be proved by projecting on the 1, 45, 210 parts and using the Jacobi identity and constraint.

It has been shown that the only independent components of the gauge field are the $\Theta=0$ components $A_{M}^{0}=\left.A_{M}\right|_{0}$ and $\Psi^{0}=\left.\Psi\right|_{0}$. All other components can be gauged away or are bosonic derivatives of the fundamental fields. The equations of motion which follow from (4.6) in much the same way as (4.7) are

$$
\begin{aligned}
{\left[\mathcal{D}_{N}, F^{N M}\right] } & =-\frac{i}{2} \Sigma_{A B}^{M}\left\{\Psi^{A}, \Psi^{B}\right\} \\
\Sigma_{A B}^{M}\left[\mathcal{D}_{M}, \Psi^{B}\right] & =0,
\end{aligned}
$$

their $\Theta=0$ part forces the fundamental fields $A_{M}^{0}$ and $\Psi^{0}$ on shell.

Using the relations (4.7) one can show that the combination ${ }^{13}$

$$
\mathcal{L}=\operatorname{Tr}\left(\frac{1}{4} F^{M N} F_{M N}+\frac{1}{2} \Psi^{A} \Sigma_{A B}^{M}\left[\mathcal{D}_{M}, \Psi^{B}\right]\right)
$$

has the property that the fermionic derivative is a total bosonic derivative, $\left[D_{A}, \mathcal{L}\right]=$ $\left[\partial_{M}, B_{A}^{M}\right]$ and the action

$$
S=\left.\frac{2}{g_{\mathrm{YM}}^{2}} \int d^{D} X \mathcal{L}\right|_{0}
$$

is thus supersymmetric.

\footnotetext{
${ }^{13}$ Note: $\mathcal{L}$ is a descendant of $\operatorname{Tr} \Phi_{(m} \Phi_{n)}$.
} 


\subsection{A Harmonic Superspace}

We introduce a harmonic superspace which is adapted to the treatment of BMN operators. It consists of the usual superspace coordinates plus additional bosonic coordinates parametrising the coset $\mathrm{SO}(6) / \mathrm{SO}(4)$. It enables us to work with the $\mathrm{SO}(4) \times \mathrm{SO}(2)$ split of BMN operators while keeping $\mathrm{SO}(6)$ invariance.

First, we reduce the $D=9+1$ superspace to four dimensions, $X^{M}=\left(x^{\mu}, 0\right)$, to obtain $\mathcal{N}=4$ super Yang-Mills theory in $D=3+1$ dimensions. The vector indices $M$ split up into $\mu=0, \ldots, 3$ and $m=1, \ldots, 6$, while we keep the spinor indices $A$ (most of the time they will be suppressed in matrix notation). The six gauge fields corresponding to the reduced coordinates, $A_{m}$, become the six scalar fields $\Phi_{m}=A_{m}$ of $\mathcal{N}=4 \mathrm{SYM}$. The field strengths with internal indices are $F_{\mu m}=\mathcal{D}_{\mu} \Phi_{m}$ and $F_{m n}=i\left[\Phi_{m}, \Phi_{n}\right]$.

In addition to the superspace coordinates $z=\left(x^{\mu}, \Theta^{A}\right)$. We introduce a complex vector $V^{m}$ in the internal space with zero square and unit norm,

$$
V^{2}=0, \quad|V|^{2}=1
$$

for example $V=\left(0,0,0,0, \frac{1}{\sqrt{2}}, \frac{i}{\sqrt{2}}\right)^{14}$. It has the remarkable property that a product of $J$ vectors $V^{m} V^{n} \ldots$ is not only completely symmetric in all indices, but also completely traceless. Therefore, the product transforms in the irreducible representation $[0, J, 0]$ of $\mathrm{SO}(6)$.

Assume $A_{m n \ldots}$ is a symmetric-traceless tensor. Then $A$ can be written as a holomorphic function in $V$

$$
A(V)=A_{m n \ldots} V^{m} V^{n} \ldots
$$

This map is injective, the components of the tensor $A$ correspond to the amplitude of certain harmonics on the space $\mathrm{SO}(6) / \mathrm{SO}(4)$, the space of $V$ s. The vacuum and $\mathrm{BMN}$ operators transform like $A$ under $\mathrm{SO}(6)$ and it is very convenient to write down these operators as holomorphic functions in $V$. This keeps $\mathrm{SO}(6)$ covariance manifest without making use of indices. In this sense, the null-vector $V$ is a set of additional bosonic coordinates of a 'harmonic' superspace. It is adapted to the $\mathrm{SO}(2) \times \mathrm{SO}(4)$ split of BMN operators in that $\mathrm{SO}(2)$ acts as a complex phase rotation on $V$ and $\mathrm{SO}(4)$ leaves $V$ invariant.

With $V$ we can isolate two components, $a_{+}$and $a_{-}$, of an internal space vector $a_{m}$

$$
a_{+}=a^{-}=a_{m} V^{m}, \quad a_{-}=a^{+}=a_{m} \bar{V}^{m}, \quad a_{i} V^{i}=a_{i} \bar{V}^{i}=0,
$$

the remaining four components, $a_{i}$, are labelled by $i=1, \ldots, 4$. The internal space metric becomes

$$
\delta_{i=j}=\delta_{+-}=\delta_{-+}=1, \quad \delta_{i \neq j}=\delta_{i+}=\delta_{i-}=\delta_{++}=\delta_{--}=0 .
$$

and the product of two $\Sigma$ matrices in the direction of $V$ vanishes

$$
\Sigma_{+} \tilde{\Sigma}_{+}=\Sigma_{++}+\delta_{++}=0
$$

\footnotetext{
${ }^{14}$ One could also set $V=\left(\tau_{1}, \tau_{2}, \tau_{3}, \tau_{4}, \frac{1}{\sqrt{2}}\left(1-\frac{1}{2} \tau^{2}\right), \frac{i}{\sqrt{2}}\left(1+\frac{1}{2} \tau^{2}\right)\right)$ with four parameters $\tau_{k}$. One can then define $\mathrm{SO}(6)$ primary fields in much the same way as conformal primary fields 14. The conjugate $\bar{V}$ is obtained by $\tau$ inversion as $V(-1 / \tau)=-\bar{V}(\tau) / \tau^{2}$ and $\mathrm{SO}(6)$ descendants are obtained by acting with $\partial / \partial \tau_{k}$. The $\mathrm{SO}(6)$ correlator of unit dimension is $V_{m}(\tau) V^{m}\left(\tau^{\prime}\right)=-\frac{1}{2}\left(\tau-\tau^{\prime}\right)^{2}$.
} 
because two + indices can neither be antisymmetrised nor do they have a trace. Using this property it is easily seen that $\tilde{P}_{+}=\frac{1}{2} \tilde{\Sigma}_{-} \Sigma_{+}, \tilde{P}_{-}=\frac{1}{2} \tilde{\Sigma}_{+} \Sigma_{-}$are orthogonal projection operators which project to one half of the spinor space.

We decompose the $\mathrm{SO}(6)$ vector of scalar fields into

$$
Z=\Phi_{+}, \quad \bar{Z}=\Phi_{-}, \quad \phi_{i}=\Phi_{i},
$$

and the spinor field into the two spinor subspaces

$$
\psi=\tilde{P}_{+} \Psi, \quad \bar{\psi}=\tilde{P}_{-} \Psi
$$

We will be interested in supersymmetry transformations that leave the number of charge defects invariant. This can be done by using the combination

$$
\delta_{\epsilon}=\epsilon^{\top} \tilde{\Sigma}_{+} \mathcal{D}=\epsilon_{A} \tilde{\Sigma}_{+}^{A B} \mathcal{D}_{B}
$$

where $\epsilon_{A}$ is an arbitrary anticommuting spinor. The matrix $\tilde{\Sigma}_{+}$projects out the parts that would increase the number of defects by one. This leaves only 8 independent variations parametrised by $\epsilon$. It commutes with the combination $\Delta-J$

$$
[\Delta-J, \delta]=0
$$

of the generator of dilatations and $\mathrm{SO}(2)$ rotations, $\Delta$ and $J$. It thus leaves the number of impurities invariant. In plane-wave string theory it corresponds to the supersymmetry generator commonly denoted by $Q^{-}$. It is easily seen that the operators $\delta$ commute among each other

$$
\left[\delta_{1}, \delta_{2}\right]=\epsilon_{1, A} \tilde{\Sigma}_{+}^{A B}\left\{\mathcal{D}_{B}, \mathcal{D}_{C}\right\} \tilde{\Sigma}_{+}^{C D} \epsilon_{2, D}=2 \epsilon_{1}^{\top} \tilde{\Sigma}_{+} \Sigma^{M} \tilde{\Sigma}_{+} \epsilon_{2} \mathcal{D}_{M}=4 i \epsilon_{1}^{\top} \tilde{\Sigma}_{+} \epsilon_{2} Z
$$

up to a gauge transformation proportional to the field $Z$. The algebra generated by the operators in (4.18) is thus a $\mathbb{R}^{8}$ subalgebra of $\mathrm{SU}(2,2 \mid 4)$. We can therefore act with the same $\delta_{\epsilon}$ iteratively to obtain all descendants of a gauge invariant operator. With the 8 independent fermionic generators $2^{8}=256$ descendants are generated unless any of the variations $\delta^{l}, l \leq 8$, annihilates the operator ${ }^{15}$. This, for example, happens for the primary vacuum operator. Let us write down the variations of the component fields using the supersymmetry rules (4.7)

$$
\begin{aligned}
\delta Z & =0 \\
\delta \psi & =-\tilde{\Sigma}_{-} \Sigma_{+} \tilde{\Sigma}^{\mu} \epsilon \mathcal{D}_{\mu} Z-i \tilde{\Sigma}_{-} \Sigma_{+} \tilde{\Sigma}^{i} \epsilon\left[\phi_{i}, Z\right] \\
\delta \phi_{i} & =\epsilon^{\top} \tilde{\Sigma}_{+} \Sigma^{i} \psi \\
\delta \mathcal{D}_{\mu} & =i \epsilon^{\top} \tilde{\Sigma}_{+} \Sigma^{\mu} \psi \\
\delta \bar{\psi} & =-i \tilde{\Sigma}_{+} \epsilon[\bar{Z}, Z]-\frac{i}{2} \tilde{\Sigma}_{+} \tilde{\Sigma}^{i j} \epsilon\left[\phi_{i}, \phi_{j}\right]-\tilde{\Sigma}_{+} \Sigma^{\mu} \tilde{\Sigma}^{i} \epsilon \mathcal{D}_{\mu} \phi_{i}-\frac{1}{2} \tilde{\Sigma}_{+} \Sigma^{\mu \nu} \epsilon F_{\mu \nu}, \\
\delta \bar{Z} & =2 \epsilon^{\top} \bar{\psi}
\end{aligned}
$$

\footnotetext{
${ }^{15}$ For each of these 256 operators 256 independent operators can be generated by the 8 generators $\epsilon^{\top} \tilde{\Sigma}_{-} \mathcal{D}$ resulting in a long supermultiplet of $2^{16}$.
} 


\subsection{BMN Operators}

In the harmonic superspace notation the primary vacuum (3.1) and BMN operators (3.3) at finite $J$ become

$$
\begin{aligned}
& \mathcal{Q}^{J}(z, V)=\frac{N_{0}^{-J}}{\sqrt{J}} \operatorname{Tr} Z^{J} \\
& \mathcal{O}_{n}^{J}(z, V)=\frac{N_{0}^{-J-2}}{\sqrt{J+3}}\left[\frac{1}{2} \sum_{p=0}^{J} \cos \frac{\pi n(2 p+3)}{J+3} \operatorname{Tr} \phi_{i} Z^{p} \phi_{i} Z^{J-p}-2 \cos \frac{\pi n}{J+3} \operatorname{Tr} \bar{Z} Z^{J+1}\right]
\end{aligned}
$$

The first important point to notice is that the variation of $Z$ vanishes (4.21). Thus the variations act only on the impurities, the string of $Z \mathrm{~s}$ in the operators appears merely as a background. The immediate consequence is that the vacuum operator is invariant under this variation

$$
\delta \mathcal{Q}^{J}=0
$$

This is the $\frac{1}{2}$ BPS condition.

It is a matter of patience and Fierz identities to work out the variations of the BMN operators

$$
\begin{aligned}
\delta^{2} \mathcal{O}_{n}^{J} & =\sqrt{2} \kappa_{n}^{J} e^{i j} \mathcal{O}_{[i j], n}^{J,(1)}+\ldots \\
\delta^{4} \mathcal{O}_{n}^{J} & =-6\left(\kappa_{n}^{J}\right)^{2} e^{i \mu} e^{j}{ }_{\mu} \mathcal{O}_{(i j), n}^{J,(2)}+\ldots
\end{aligned}
$$

This involves the combination of two variation coefficients ${ }^{16}$

$$
e_{M N}=\epsilon^{\top} \tilde{\Sigma}_{+} \Sigma_{M N} \epsilon
$$

and a constant

$$
\kappa_{n}^{J}=\sqrt{8} N_{0} \sin \frac{\pi n}{J+3}=\sqrt{8} \sqrt{\frac{g_{\mathrm{YM}}^{2} N}{8 \pi^{2}}} \sin \frac{\pi n}{J+3} \sim \sqrt{\lambda^{\prime}} n .
$$

The supersymmetry variation (4.24) is the principal result of this section. It shows that the operators

$$
\begin{gathered}
\mathcal{O}_{[i j], n}^{J,(1)}(z, V)=\frac{N_{0}^{-J-3}}{\sqrt{J+3}} \sum_{p=0}^{J+1} i \sin \frac{\pi n(2 p+2)}{J+3} \operatorname{Tr} \phi_{[i} Z^{p} \phi_{j]} Z^{J+1-p}, \\
\mathcal{O}_{(i j), n}^{J,(2)}(z, V)=\frac{N_{0}^{-J-4}}{\sqrt{J+3}} \sum_{p=0}^{J+2} \cos \frac{\pi n(2 p+1)}{J+3} \operatorname{Tr} \phi_{(i} Z^{p} \phi_{j)} Z^{J+2-p},
\end{gathered}
$$

belong to the same supermultiplet as the primary operator $\mathcal{O}_{n}^{J}$. This proves that they have degenerate anomalous dimension to all orders in perturbation theory (see also the

\footnotetext{
${ }^{16}$ The variation coefficients allow one to project the most general descendant operator to one specific operator. For example, the combination $\left(\partial_{\epsilon}^{\top} \Sigma_{-} \tilde{\Sigma}_{i j} \partial_{\epsilon}\right)$ projects the general variation $\delta^{2} \mathcal{O}_{n}^{J}$ to the operator $\mathcal{O}_{n,[i j]}^{J,(2)}$ with the coefficient $e_{i j}$ due to the identity $\left(\partial_{\epsilon}^{\top} \Sigma_{-} \tilde{\Sigma}_{i j} \partial_{\epsilon}\right) e^{M N} \sim \delta_{[i}^{M} \delta_{j]}^{N}$.
} 
discussion at the end of Sec. 3). Furthermore the opertators coincide with the other two operators we obtained by a direct calculation in (3.3). We have presented only the descendants with two scalar impurities here, the complete set of bosonic descendants can be found in App. B. It verifies the claim of Sec. 2 that all a BMN multiplet contains all flavours of BMN operators.

The constants appearing in front of the operators were chosen such that the two-point function of these operators are canonically normalised as in (3.7). The constant $\kappa_{n}^{J}$ is in fact the square root of the anomalous dimension. Its appearance is related to a splitting of the long multiplet at $g_{\mathrm{YM}}=0$ which happens at the unitarity bound of the A series of unitary irreducible representations of $\mathrm{SU}(2,2 \mid 4)$ [33.

We can also write the above variations in a general form as

$$
\delta^{l} \mathcal{O}_{n}^{J}=\sum_{a} N_{a, n}^{J,(l / 2)}\left(\epsilon^{l}\right)^{a} \mathcal{O}_{a, n}^{J,(l / 2)}
$$

Here, a labels the descendant operators at level $l,\left(\epsilon^{l}\right)^{a}$ is the corresponding variation coefficient and $N_{a, n}^{J,(l / 2)}$ is a normalisation constant. To be explicit we write down these quantities for the operators in (4.24)

$$
\begin{array}{ll}
N_{[i j], n}^{J,(1)}=\sqrt{2} \kappa_{n}^{J}, & \left(\epsilon^{2}\right)^{[i j]}=e^{i j} \\
N_{(i j), n}^{J,(2)}=-6\left(\kappa_{n}^{J}\right)^{2}, & \left(\epsilon^{4}\right)^{(i j)}=e^{i \mu} e_{\mu}^{j}
\end{array}
$$

\subsection{Operator Mixing}

When considering non-planar corrections to correlation functions one has to take into account that operators with different numbers of traces undergo mixing. The fact that different BMN operators are related to another by supersymmetry means that the mixing matrices are also related. We will now investigate this relationship.

In 21,22] the issue of operator mixing was investigated in the BMN limit up to the torus and up to one-loop. The resulting expressions for the modified operators at $\mathcal{O}\left(g_{2}\right)$ $\operatorname{are}^{17}$

$$
\begin{aligned}
\mathcal{O}_{n}^{\prime J} & =\mathcal{O}_{n}^{J}-g_{2} \sum_{k, r} n^{2} X_{n, k}^{J, r} \mathcal{O}_{k}^{J r} \mathcal{Q}^{J(1-r)}-g_{2} \sum_{r} n^{2} Y_{n}^{J, r} \frac{1}{2} \mathcal{Q}_{i}^{J r+1,[1]} \mathcal{Q}_{i}^{J(1-r)+1,[1]} \\
\mathcal{O}_{[i j], n}^{\prime J,(1)} & =\mathcal{O}_{n}^{J}-g_{2} \sum_{k, r} n \frac{k}{r} X_{n, k}^{J, r} \mathcal{O}_{[i j], k}^{J r,(1)} \mathcal{Q}^{J(1-r)} \\
\mathcal{O}_{(i j), n}^{\prime J,(2)} & =\mathcal{O}_{n}^{J}-g_{2} \sum_{k, r} \frac{k^{2}}{r^{2}} X_{n, k}^{J, r} \mathcal{O}_{(i j), k}^{J r,(2)} \mathcal{Q}^{J(1-r)} \\
X_{n, k}^{J, r} & =\frac{\sqrt{1-r} \sin ^{2}(\pi n r)}{\sqrt{J r}\left(n^{2}-\frac{k^{2}}{r^{2}}\right)^{2}}, \quad Y_{n}^{J, r}=-\frac{\sin ^{2}(\pi n r)}{\sqrt{J} \pi^{2} n^{2}}
\end{aligned}
$$

These three expressions are very similar to each other. When going from the singlet to the antisymmetric to the symmetric-traceless operator the coefficient in front of $\mathcal{O}_{k}^{J r} \mathcal{Q}^{J(1-r)}$ is

\footnotetext{
${ }^{17}$ The expressions found in 21 had to be (anti)symmetrised in the mode numbers for the singlet and (anti)symmetric operators to project to the relevant part of the mixing matrix.
} 
multiplied by $k / n r$ and the operator $\mathcal{Q}_{i}^{J r+1,[1]} \mathcal{O}_{j}^{J(1-r)+1,[1]}$ is dropped. This pattern is due to supersymmetry. We apply $\delta^{2}$ to the right hand side of the first equation of (4.30) and find that the single-trace operator gets multiplied by the normalisation constant $N_{[i j], n}^{J,(1)}$ while the first double-trace operator gets multiplied by $N_{[i j], k}^{J r,(1)}$. The second double-trace operator does not have an $e^{i j}$ descendant and drops out. The resulting expression is to be compared to the second line in (4.30). It is easily seen that the expressions match provided that $\delta^{2} \mathcal{O}_{n}^{\prime J}=N_{[i j], n}^{J,(1)} e^{i j} \mathcal{O}_{[i j], n}^{J,(1)}+\ldots$, i.e. the same variation as for $\mathcal{O}_{n}^{J}$, in (4.24). Effectively this means that only the coefficient of the double-trace operator changes by $N_{[i j], k}^{J r,(1)} / N_{[i j], n}^{J,(1)} \sim k / n r$. A similar discussion applies to the third operator in (4.30) and also to the mixing matrix of double-trace operators. It is interesting to compare the mixing of BMN operators at large $J$ and small $J$. It can be seen by comparing to [40,41,15] that the mixing pattern of single-trace and double-trace operators is essentially the same as in the BMN limit, 4.30) 21.

The conclusion is that the variations of the redefined operators do not change at $\mathcal{O}\left(g_{2}\right)$. Using the expressions and matrices in [21] we find that the normalisation constants of the variations do change at $\mathcal{O}\left(g_{2}^{2}\right)$, while the form of the variations (4.28) remains unchanged due to nontrivial relations between the matrices. The normalisation constants on the torus are

$$
\begin{aligned}
& N_{[i j], n}^{J,(1)} \sim \sqrt{2 \lambda^{\prime}} n\left(1+g_{2}^{2}\left(\frac{1}{96 \pi^{2} n^{2}}+\frac{35}{256 \pi^{4} n^{4}}\right)\right), \\
& N_{(i j), n}^{J,(2)} \sim-6 \lambda^{\prime} n^{2}\left(1+g_{2}^{2}\left(\frac{1}{48 \pi^{2} n^{2}}+\frac{35}{128 \pi^{4} n^{4}}\right)\right) .
\end{aligned}
$$

As we shall see later this modification is related to a modification of the anomalous dimension on the torus. In the following we shall consider only the full operators after a complete normalisation and diagonalisation. Certainly, this can be done in a perturbative fashion and the operators as defined in (3.3) are just their lowest-order approximations.

\section{Correlation functions}

In this section we will consider correlation functions involving BMN operators and restrictions on their form imposed by superconformal symmetry. The aim is to obtain the form of correlation functions in superspace and apply it to relate correlators of BMN operators which have been calculated [21,22, We will start by reviewing some results of $\mathcal{N}=4$ superconformal symmetry and later apply them to correlators of BMN operators.

\subsection{Review of Superconformal Symmetry}

Conformal symmetry in four spacetime dimensions has been addressed in the works [42, 43, 44, 45] with different numbers of supersymmetries. We will start by reviewing some results of 45 in the notation of that paper.

In this context it is useful to consider $\mathcal{N}=4$ superspace as a coset space of the supergroup $\mathrm{SU}(2,2 \mid 4)$ over the subgroup generated by spacetime rotations, internal rotations, dilatations and superconformal boosts. Due to this coset space construction it 
is natural to believe that $\mathcal{N}=4$ superspace is not flat, i.e. tangent vectors at different points in superspace cannot be compared directly. For correlators of operators with a tensor structure, however, this is exactly what one needs to do. The tensor indices at one point in superspace need to be saturated by tensor indices at some other point.

In [45] expressions for the connection of $\mathrm{SU}(4)$ internal and $\mathrm{SL}(2, \mathbb{C})$ spacetime spinors were given

$$
\hat{v}_{b}^{a}\left(z_{12}\right)=\left(\frac{x_{\overline{2} 1}^{2}}{x_{\overline{1} 2}^{2}}\right)^{1 / 4} v_{b}^{a}\left(z_{12}\right) \in \mathrm{SU}(4), \quad \hat{\tilde{x}}_{\overline{1} 2}=\frac{\tilde{x}_{\overline{1} 2}}{\left|x_{\overline{1} 2}\right|} \in \mathrm{SL}(2, \mathbb{C})
$$

where

$$
v_{b}^{a}\left(z_{12}\right)=\delta_{b}^{a}+4 i \theta_{12}^{a} \tilde{x}_{\overline{1} 2}^{-1} \bar{\theta}_{12 b}
$$

Using the invariant tensors $\sigma_{a b}^{m}$ an $\mathrm{SO}(6)$ vector index can be transformed into two antisymmetric $\mathrm{SU}(4)$ spinor indices. In this way internal vectors at two different points in superspace can be related with the connection

$$
J_{12, m n}=\frac{1}{4}\left(\frac{x_{\overline{2} 1}^{2}}{x_{\overline{1} 2}^{2}}\right)^{1 / 2} \sigma_{m, a b} v_{c}^{a}\left(z_{12}\right) v_{d}^{b}\left(z_{12}\right) \sigma_{n}^{c d} \in \mathrm{SO}(6) .
$$

Due to the self-duality of the 6 representation of $\mathrm{SU}(4)$ the internal vector connection can also be written in a different fashion

$$
J_{12, m n}=\frac{1}{4}\left(\frac{x_{\overline{1} 2}^{2}}{x_{\overline{2} 1}^{2}}\right)^{1 / 2} \sigma_{n, a b} v_{c}^{a}\left(z_{21}\right) v_{d}^{b}\left(z_{21}\right) \sigma_{m}^{c d} \in \mathrm{SO}(6)
$$

Equivalently, for spacetime vectors there is the connection

$$
J_{12, \mu \nu}=\frac{1}{2} \operatorname{Tr} \sigma_{\mu} \hat{\tilde{x}}_{\overline{1} 2} \sigma_{\nu} \hat{\tilde{x}}_{\overline{2} 1}=\frac{1}{2} \operatorname{Tr} \tilde{\sigma}_{\mu} \hat{x}_{\overline{2} 1} \tilde{\sigma}_{\nu} \hat{x}_{\overline{1} 2}
$$

The $\theta=\bar{\theta}=0$ components of $P_{12}, J_{12, m n}$ and $J_{12, \mu \nu}$ are given by

$$
\left.P_{12}\right|_{0}=\frac{1}{\left(x_{1}-x_{2}\right)^{2}},\left.\quad J_{12, m n}\right|_{0}=\delta_{m n},\left.\quad J_{12, \mu \nu}\right|_{0}=\eta_{\mu \nu}-2 \frac{\left(x_{1}-x_{2}\right)_{\mu}\left(x_{1}-x_{2}\right)_{\nu}}{\left(x_{1}-x_{2}\right)^{2}} .
$$

In correlation functions internal space vectors are usually due to the field $\Phi_{m}$ of unit dimension. Therefore one is tempted to combine $J_{12, m n}$ with the superconformal scalar correlator of unit dimension

$$
P_{12}=\frac{1}{\left(x_{\overline{1} 2}^{2} x_{\overline{2} 1}^{2}\right)^{1 / 2}}
$$

to

$$
K_{12, m n}=P_{12} J_{12, m n}=\frac{\sigma_{m, a b} v^{a}{ }_{c}\left(z_{12}\right) v_{d}^{b}\left(z_{12}\right) \sigma_{n}^{c d}}{4 x_{\overline{1} 2}^{2}}=\frac{\sigma_{n, a b} v_{c}^{a}\left(z_{21}\right) v_{d}^{b}\left(z_{21}\right) \sigma_{m}^{c d}}{4 x_{\overline{2} 1}^{2}} .
$$

This is the unique two-point function of unit conformal dimension at points $z_{1}$ and $z_{2}$ which relates an internal vector index $m$ at point $z_{1}$ with the index $n$ at point $z_{2}$ in a superconformally covariant way. An interesting feature of the first form of the function 
$K_{12, m n}$ is that it depends only on the coordinates $x_{\overline{1} 2}$ and $\bar{\theta}_{12}$, which are anti-chiral at point $z_{1}$. The only exception is a chiral $\theta_{12}^{a}$ in $v^{a}{ }_{b}\left(z_{12}\right)$, (5.2), a chiral derivative $D_{1}$ with respect to $z_{1}$ will therefore act only on this. Now assume that we act with the combination $V^{p} \sigma_{p}^{b a} D_{1, a \alpha}$ on $V^{m} K_{12, m n}$ where $V$ is a (complex) null-vector of $\mathrm{SO}(6)$. We then find that the result is proportional to $V^{p} \sigma_{p}^{b a} V^{m} \sigma_{m, a c}=V^{2} \delta_{c}^{b}=0$, i.e.

$$
\left(V^{p} \sigma_{p}^{b a} D_{1, a \alpha}\right)\left(V^{m} K_{12, m n}\right)=0 \text { for } V^{2}=0 .
$$

A similar argument holds for the second form of $K$ in (5.8) and anti-chiral derivatives

$$
\left(V^{p} \sigma_{p, b a} \bar{D}_{1, \alpha}^{a}\right)\left(V^{m} K_{12, m n}\right)=0 \text { for } V^{2}=0 .
$$

This remarkable property shows that $V^{m} K_{12, m n}$ is invariant under half the supersymmetry. We will make excessive use of it in the context of the vacuum operators which are $\frac{1}{2}$ BPS.

Two-point functions of superconformal (quasi)primary operators are uniquely determined by the representation and conformal dimension of the operator. To construct a two-point function the representations of the spacetime and internal group have to be transformed into a tensor product of spinor representations, The spinor indices are then contracted by the $\mathrm{SL}(2, \mathbb{C})$ or $\mathrm{SU}(4)$ connections (5.1). This is to be multiplied by $P_{12}^{\Delta}$, where $\Delta$ is the scaling dimension of the operator. We are dealing only with operators in trivial spacetime representations and tensor product representations of $\mathrm{SO}(6)$ vectors. Therefore, the vector indices can be saturated by powers of $K_{12, m n}$ and the remaining scaling dimension by powers of $P_{12}$.

Superconformal symmetry does not determine three-point functions of superconformal primary operators uniquely. There are, however, some principles which constrain their form. First of all the conformal dimension at each of the three points must match the dimension of the operator at that point. This can always be achieved by products of scalar propagators $P_{12}, P_{23}$ and $P_{31}$. Secondly, the indices of the operators need to be connected to each other in a covariant way. The most general way to accomplish this is to parallel transport the indices of two operators to the point of the third one. At that point the indices can be contracted with each other or with a covariant superspace tangent vector, $\mathbb{Z}$. We will, however, proceed differently. We construct three-point functions as products of two-point functions between all three pairs of points. Examples of this are given in [46, 47]. It is not clear to the author whether this is as general as the above construction, nevertheless, there is reasonable freedom as we shall see below. We will use the above construction rules as a guideline to derive possible three-point functions of the vacuum and BMN operators. This will then turn out to reproduce explicitly computed correlators exactly.

An interesting feature of three-point functions is that two connections can be joined to obtain a different connection from the direct one, e.g.

$$
v^{a}{ }_{b}\left(z_{12}\right) v^{b}{ }_{c}\left(z_{23}\right) \neq v^{a}{ }_{c}\left(z_{13}\right) \text {. }
$$

This is not possible for two-point functions, as there are only two points and for $z_{3}=z_{1}$ this combination is the unit matrix. One can furthermore construct longer chains of connections which can either end in indices of the operators or be closed. A closed chain turns out to be a combination of the two $\mathcal{N}=4$ superconformal three-point invariants. 


\subsection{Two-Point Functions}

With these results we consider two-point functions of BMN operators in the reduced ten-dimensional notation of the last section (see also App. A). In this notation the supertranslation invariant interval $z_{12}=\left(x_{12}^{\mu}, \Theta_{12}^{A}\right),\left(Q_{1}+Q_{2}\right) z_{12}=0$, is

$$
x_{12}^{\mu}=x_{1}^{\mu}-x_{2}^{\mu}-\Theta_{1}^{\top} \Sigma^{\mu} \Theta_{2}, \quad \Theta_{12}^{A}=\Theta_{1}^{A}-\Theta_{2}^{A} .
$$

The combination $x_{\overline{12}}$ of 45 ] corresponds to

$$
y_{12}^{\mu}=x_{12}^{\mu}-\frac{i}{12} \varepsilon^{\mu \nu \rho \sigma} \Theta_{12}^{\top} \Sigma_{\nu \rho \sigma} \Theta_{12}=x_{12}^{\mu}+\frac{i}{2} \Theta_{12}^{\top} \Sigma_{(4)} \Sigma^{\mu} \Theta_{12} \text {, }
$$

in our language, where $\Sigma_{(4)}=\frac{1}{24} \varepsilon^{\mu \nu \rho \sigma} \Sigma_{\mu \nu \rho \sigma}$. Its conjugate $\bar{y}_{12}=x_{12}^{\mu}-\frac{i}{2} \Theta_{12}^{\top} \Sigma_{(4)} \Sigma^{\mu} \Theta_{12}$ corresponds to $-x_{\overline{2} 1}$.

With these we can construct the scalar propagator

$$
P_{12}=\frac{1}{\left(y_{12}^{2} \bar{y}_{12}^{2}\right)^{1 / 2}}
$$

of unit dimension at points $z_{1}$ and $z_{2}$. The propagator of unit dimension correlating two $\mathrm{SO}(6)$ vectors is some function $K_{12, m n}$. It has the property that the variations

$$
\delta_{1}=V_{1}^{m} \epsilon_{1}^{\top} \tilde{\Sigma}_{m} D_{1}, \quad \delta_{2}=V_{2}^{m} \epsilon_{2}^{\top} \tilde{\Sigma}_{m} D_{2}
$$

annihilate the combinations

$$
\delta_{1}\left(V_{1}^{m} K_{12, m n}\right)=0, \quad \delta_{2}\left(V_{2}^{n} K_{12, m n}\right)=0 .
$$

This is all we need to know about it. Superconformally covariant two-point functions of operators involving only internal vector indices can be constructed from these two building blocks.

Superconformal symmetry fixes two-point functions of superconformal (quasi)primary operators uniquely. The operators $\mathcal{Q}^{J}$ and $\mathcal{O}_{n}^{J}$ are superconformal primaries, essentially because they cannot be a descendant of any operator, see the discussion in Sec. 2. We construct a conformally covariant two-point function for the vacuum operator $\mathcal{Q}^{J}$ from the above building blocks. Its $\mathrm{SO}(6)$ representation is $[0, J, 0]$, the symmetric-traceless tensor product of $J$ vectors. The scaling dimension $\Delta=J$ is protected from acquiring quantum corrections. Consequently, the unique two-point function is

$$
\left\langle\mathcal{Q}^{J}\left(z_{1}, V_{1}\right) \mathcal{Q}^{J}\left(z_{2}, V_{2}\right)\right\rangle=K_{12}^{J}
$$

with

$$
K_{12}=V_{1}^{m} V_{2}^{n} K_{12, m n} .
$$

Here we use independent null-vectors $V_{1}, V_{2}$ to be able to project each operator to an arbitrary component of the $\mathrm{SO}(6)$ multiplet. To compare to explicit computations we set $V_{1}=\bar{V}_{2}$, because $\mathcal{Q}(z, \bar{V})=\mathcal{Q}(z, V)^{*}$. Eq. (5.16) implies that

$$
\delta_{1}\left\langle\mathcal{Q}^{J}\left(z_{1}, V_{1}\right) \mathcal{Q}^{J}\left(z_{2}, V_{2}\right)\right\rangle=\delta_{2}\left\langle\mathcal{Q}^{J}\left(z_{1}, V_{1}\right) \mathcal{Q}^{J}\left(z_{2}, V_{2}\right)\right\rangle=0
$$


reflecting the $\frac{1}{2}$ BPS property $\delta \mathcal{Q}^{J}=0$.

In the case of the BMN operators $\mathcal{O}_{n}^{J}$ there are $J$ indices from the representation $[0, J, 0]$ to be connected in just the same way as for the vacuum operators. The conformal dimension, however, is not saturated by this, we need to multiply by powers of $P_{12}$ to match it. The correlator is thus

$$
\left\langle\mathcal{O}_{n}^{J}\left(z_{1}, V_{1}\right) \mathcal{O}_{n}^{J}\left(z_{2}, V_{2}\right)\right\rangle=K_{12}^{J} P_{12}^{\Delta_{n}^{J}-J}
$$

Again the combination $K_{12}$ is invariant under $\delta_{1}, \delta_{2}$ and just like the string of $Z \mathrm{~s}$ in the definition of the operators (3.3) it may be viewed as a background. What remains is the propagator of a Konishi-field (with modified anomalous dimension). The BMN operators could therefore be viewed as Konishi operators in a background that provides large dimension and charge.

We now perform a direct calculation of some descendant correlators. To this end we need to consider only the $P_{12}$ part of the two-point function as $K_{12}$ is invariant under the variations. First we work out the variation on $y_{12}$

$$
\delta_{1} y_{12}^{\mu}=2 \epsilon_{1}^{\top} \tilde{\Sigma}_{+} P \Sigma^{\mu} \Theta_{12}
$$

where $P=\frac{1}{2}+\frac{i}{2} \Sigma_{(4)}$ is a chiral projector. A double variation $\delta_{1}^{2}$ on $y_{12}$ vanishes due to two $\Sigma_{+}$colliding. As we will be projecting to the $\Theta=0$ component in the end the resulting $\Theta_{12}$ from the variation of $y_{12}$ must be compensated by the action of $\delta_{2}$. The only relevant variation is thus

$$
e_{12}^{\mu}=\delta_{1} \delta_{2} y_{12}^{\mu}=-2 \epsilon_{1}^{\top} \tilde{\Sigma}_{+} P \Sigma^{\mu} \tilde{\Sigma}_{-} \epsilon_{2}
$$

We can introduce an effective variation $\delta$ acting on $y_{12}$ by

$$
\delta y_{12}^{\mu}=e_{12}^{\mu}, \quad \delta \bar{y}_{12}^{\mu}=\bar{e}_{12}^{\mu},
$$

with $\bar{e}_{12}^{\mu}=-2 \epsilon_{1}^{\top} \tilde{\Sigma}_{+} \bar{P} \Sigma^{\mu} \tilde{\Sigma}_{-} \epsilon_{2}$. For $l$ consecutive variations we have to make sure the effective variation produces the right combinatorial factors. All variations on $z_{1}$ can be performed first, this yields $l$ powers of $\Theta_{12}$. The variations on $z_{2}$ should later on annihilate all these and there are $l$ ! equivalent ways to do so, thus we get

$$
\left.\left(\delta_{1} \delta_{2}\right)^{l} F\left(z_{12}\right)\right|_{0}=\left.l ! \delta^{l} F\left(z_{12}\right)\right|_{0} .
$$

For the variations of the scalar correlator we find using (5.14), (5.6)

$$
\begin{aligned}
& \left.\left(\delta_{1} \delta_{2}\right)^{2} P_{12}^{\Delta}\right|_{0}=\Delta(\Delta-2) e_{1}^{i j} e_{2}^{k l} \frac{\delta_{i[k} \delta_{l] j}}{\left|x_{12}\right|^{2 \Delta+2}}+\ldots \\
& \left.\left(\delta_{1} \delta_{2}\right)^{4} P_{12}^{\Delta}\right|_{0}=9 \Delta^{2}(\Delta-2)^{2}\left(e_{1}^{i \mu} e_{1 \mu}^{j}\right)\left(e_{2}^{k \nu} e_{2 \nu}^{l}\right) \frac{\delta_{i(k} \delta_{l) j}}{\left|x_{12}\right|^{2 \Delta+4}}+\ldots
\end{aligned}
$$

for the components that correspond to BMN operators with two scalar impurities. The complete set of even variations can be found in App. C. The tensors $e_{1}^{i j}, e_{2}^{i j}$ are the $\epsilon^{2}$ 
variation coefficients at the two points $z_{1}$ and $z_{2}$, see (4.25). For the correlator of two BMN operators with $\Delta=\Delta_{n}^{J}-J$ we may write this in a general form as

$$
\left.\left(\delta_{1} \delta_{2}\right)^{l}\left\langle\mathcal{O}_{n}^{J}\left(z_{1}, V\right) \mathcal{O}_{m}^{J}\left(z_{2}, \bar{V}\right)\right\rangle\right|_{0}=\sum_{a, b}\left(N_{a, n}^{J,(l / 2)}\right)^{2}\left(\epsilon_{1}^{l}\right)^{a}\left(\epsilon_{2}^{l}\right)^{b} \frac{\delta_{n m} J_{12, a b}}{\left|x_{12}\right|^{2 \Delta_{n}^{J}+l}},
$$

similar to (4.28). Here, the symbol $J_{12, a b}$ relates the spacetime and internal indices of the operators $a$ and $b$ in a conformally covariant way, usually as a product of $J_{12, m n}=\delta_{m n}$ for $\mathrm{SO}(6)$ indices and $J_{12, \mu \nu}=\eta_{\mu \nu}-2 x_{12, \mu} x_{12, \nu} / x_{12}^{2}$ for spacetime indices. We assume the descendant operators to be normalised such that their two-point function is

$$
\left.\left\langle\mathcal{O}_{a, n}^{J,(l / 2)}\left(z_{1}, V\right) \mathcal{O}_{b, m}^{J,(l / 2)}\left(z_{2}, \bar{V}\right)\right\rangle\right|_{0}=\frac{\delta_{n m} J_{12, a b}}{\left|x_{12}\right|^{2 \Delta_{n}^{J}+l}} .
$$

Using the definition of descendant operators in (4.28) we can compare (5.26), (5.27) to (5.25) with $\Delta=\Delta_{n}^{J}-J \approx 2$ to read off the exact normalisation coefficients in terms of the scaling dimension. We obtain

$$
\begin{aligned}
& N_{[i j], n}^{J,(1)}= \pm \sqrt{\Delta_{n}^{J}-J} \sqrt{\Delta_{n}^{J}-J-2} \approx \pm \sqrt{2} \times \sqrt{8} \sqrt{\frac{g_{\mathrm{YM}}^{2} N}{8 \pi^{2}}} \sin \frac{\pi n}{J+3} \\
& N_{(i j), n}^{J,(2)}= \pm 3\left(\Delta_{n}^{J}-J\right)\left(\Delta_{n}^{J}-J-2\right) \approx \pm 6 \times 8 \frac{g_{\mathrm{YM}}^{2} N}{8 \pi^{2}} \sin ^{2} \frac{\pi n}{J+3}
\end{aligned}
$$

to be compared to (4.29). We collect all even normalisation coefficients in App. D, They can easily be seen to agree with the direct variation of the bare operators in App. B at leading order. We note that some of the normalisation constants involve factors of $\delta \Delta_{n}^{J}=\Delta_{n}^{J}-J-2$, the anomalous dimension of the BMN operators. In the direct variation of operators (4.24) these correspond to factors of $\left(\kappa_{n}^{J}\right)^{2} \approx \delta \Delta_{n}^{J}$.

Moreover, this can be extended to higher genus. Due to redefinitions of operators on the torus their variations can be altered by pieces proportional to $1 / N^{2}$, see (4.31). These changes are reflected by a shift in the scaling dimension on the torus. The anomalous dimension on the torus has been calculated in the BMN limit, see (1.2). Here, we obtain an exact normalisation constant $N_{[i j], n}^{J,(1)}=\sqrt{\left(\Delta_{n}^{J}-J\right)\left(\Delta_{n}^{J}-J-2\right)} \sim \sqrt{2 \delta \Delta_{n}^{J}}$ which agrees with (4.31). This, however, does not mean that supersymmetry determines the anomalous dimension on the torus. The crucial input of (4.31) is the mixing matrix which is the result of a $\mathcal{O}\left(g_{2}^{2} \lambda^{\prime}\right)$ calculation. Supersymmetry only yields non-trivial relations among the mixing matrix elements [21] belonging to different descendant operators.

\subsection{Three-Point Functions}

In the following we will consider the implications of superconformal symmetry on threepoint functions of vacuum and BMN operators. A few correlators of descendants of these operators were computed in the BMN limit in [11,13, 16, 21, 22. It can be observed that some of these correlators vanish and others are closely related to another. These relations will be explained. 
The form of three-point functions of primary operators is in general not completely fixed by superconformal symmetry. We will therefore have to rely on some additional constraint to fix the form, namely the $\frac{1}{2}$ BPS condition of the vacuum operators $\mathcal{Q}^{J}$. A general treatment of three-point function with some desired properties is rather involved, see e.g. [4], and we will have to make some simplifying assumptions here: The operators under consideration are singlets under the spacetime group and carry internal vector indices. Consequently we will assume that a generic three-point function can be constructed from the building blocks $P$ and $K_{m n}$ that have turned out useful before, see also [47. We will use three guiding principles in the construction. The conformal dimensions at the three points must match the dimensions of the operators, the indices must be saturated and for vacuum operators the $\frac{1}{2}$ BPS condition must hold manifestly by (5.17). With these guidelines we are able to explain the structure of the three point functions that have been worked out explicitly. The third principle, however, does not exclude the existence of a three-point function that has the $\frac{1}{2}$ BPS condition fulfilled by other means.

Three vacuum operators. To demonstrate the method we consider a three-point function of three vacuum operators [46, 47]. This three-point function is unique due to three $\frac{1}{2}$ BPS conditions to be satisfied. Effectively this means that it depends only on 24 instead of 48 fermionic coordinates. By superconformal transformations we can gauge away up to 32 fermionic coordinates, which fixes this three-point function of scalar operators uniquely [47. Due to the $\frac{1}{2}$ BPS conditions at all points the functions $K$ can only be used in the combinations $K_{12}, K_{23}, K_{31}$. Assume the charges of the operators are $J_{1}, J_{2}, J_{3}$. Then it is easily seen that $\left(J_{1}+J_{2}-J_{3}\right) / 2$ indices at point $z_{1}$ have to be connected to $z_{2}$ and so on. The only way to do this is in the combination $K_{12}$. Multiplying the other two connections we find that the dimensions of the operators match automatically. The correlator is thus

$$
\begin{aligned}
& \left\langle\mathcal{Q}^{J_{1}}\left(z_{1}, V_{1}\right) \mathcal{Q}^{J_{2}}\left(z_{2}, V_{2}\right) \mathcal{Q}^{J_{3}}\left(z_{3}, V_{3}\right)\right\rangle= \\
& C^{J_{1} J_{2} J_{3}} K_{12}^{\left(J_{1}+J_{2}-J_{3}\right) / 2} K_{23}^{\left(J_{2}+J_{3}-J_{1}\right) / 2} K_{31}^{\left(J_{3}+J_{1}-J_{2}\right) / 2} .
\end{aligned}
$$

There is one condition that the charges must satisfy, namely the powers of $K_{12}, K_{23}, K_{31}$ must be non-negative and integer [47. Otherwise the $\mathrm{SO}(6)$ indices cannot be fully saturated. Put differently, the $K \mathrm{~s}$ must yield polynomial expressions in $V_{1}, V_{2}$. We refrain from giving an explicit coefficient $C^{J_{1} J_{2} J_{3}}$ for this three-point function because the issue of diagonalisation of the vacuum sector is not settled [21]. For the bare operators and $J_{1}+J_{2}=J_{3}$ an all-genus expression is found in [11].

Two vacuum and one BMN operator. Next we consider a three-point function of two vacuum operators at points $z_{1}, z_{2}$ and one BMN operator at point $z_{3}$. Due to two $\frac{1}{2}$ BPS conditions this three-point function depends on 32 fermionic coordinates and is apparently uniquely fixed as well, see above. The $\frac{1}{2}$ BPS conditions at points $z_{1}, z_{2}$ imply that the propagators can only be used in the combinations $K_{12}, V_{1}^{m} K_{13, m p}$ and $V_{2}^{n} K_{23, n p}$. The two last combinations can be contracted with $V_{3}^{p}$ as before or they can be joined

$$
K_{132}=V_{1}^{m} K_{13, m}^{p} K_{32, p n} V_{1}^{n} .
$$


This connection is different from the direct connection $K_{12}$ in that it has conformal dimension 2 at point $z_{3}$. In total there are four building blocks for this three-point function, $K_{12}, K_{13}, K_{23}, K_{132}$ and the resulting correlator is

$$
\begin{aligned}
& \left\langle\mathcal{Q}^{J_{1}}\left(z_{1}, V_{1}\right) \mathcal{Q}^{J_{2}}\left(z_{2}, V_{2}\right) \mathcal{O}_{n}^{J_{3}}\left(z_{3}, V_{3}\right)\right\rangle= \\
& C_{n}^{J_{1} J_{2} J_{3}} K_{132}^{a} K_{12}^{b} K_{13}^{\left(J_{1}+J_{3}-J_{2}\right) / 2} K_{23}^{\left(J_{2}+J_{3}-J_{1}\right) / 2} .
\end{aligned}
$$

The numbers $a, b$ must be adjusted such that the conformal dimensions match and all $V \mathrm{~s}$ come in the right power, i.e. $a=\frac{1}{2}\left(\Delta_{n}^{J_{3}}-J_{3}\right), b=\frac{1}{2}\left(J_{1}+J_{2}-\Delta_{n}^{J_{3}}\right)$. Three correlators of this form have been calculated for descendant operators in the BMN limit and at leading order [13, 16, 21, 22

$$
\begin{aligned}
& \left\langle\mathcal{Q}_{i}^{J_{1},[1]}\left(z_{1}, V\right) \mathcal{Q}_{j}^{J_{2},[1]}\left(z_{2}, V\right) \mathcal{O}_{n}^{J_{3}}\left(z_{3}, \bar{V}\right)\right\rangle=0, \quad J_{3}=J_{1}+J_{2}-2, \\
& \left\langle\mathcal{Q}_{i}^{J_{1},[1]}\left(z_{1}, V\right) \mathcal{Q}_{j}^{J_{2},[1]}\left(z_{2}, V\right) \mathcal{O}_{[k l], n}^{J_{3},(1)}\left(z_{3}, \bar{V}\right)\right\rangle=0, \quad J_{3}=J_{1}+J_{2}-3, \\
& \left\langle\mathcal{Q}_{i}^{J_{1},[1]}\left(z_{1}, V\right) \mathcal{Q}_{j}^{J_{2},[1]}\left(z_{2}, V\right) \mathcal{O}_{(k l), n}^{J_{3},(2)}\left(z_{3}, \bar{V}\right)\right\rangle \neq 0, \quad J_{3}=J_{1}+J_{2}-4 .
\end{aligned}
$$

The second zero is readily explained, $J_{1}+J_{2}+J_{3}$ is odd and therefore the $\mathrm{SO}(6)$ indices cannot be contracted. The first zero could be explained by demanding that all $K$ come in non-negative powers. Negative powers of $K$ would give rise to non-polynomial expressions in the $V$ s. Strictly speaking, this is not a problem here, because $K_{12}$ and $K_{132}$ are both proportional to $V_{1} \cdot V_{2}$ at $\Theta=0$. Their product is a positive integer power of $V_{1} \cdot V_{2}$ plus fermionic (nilpotent) corrections and thus still polynomial. Nevertheless we will use this positivity bound as a guiding principle in the construction of further three-point functions. It appears to give satisfactory results here, but it may be a wrong assumption in general. Positivity would imply

$$
J_{1}+J_{2} \geq \Delta_{n}^{J_{3}}
$$

If this is not satisfied, the correlator should vanish.

One vacuum and two BMN operators. The final and most interesting three-point function is a correlator of two BMN operators at $z_{1}, z_{2}$ and one vacuum operator at $z_{3}$. The single $\frac{1}{2}$ BPS condition is not enough to fix the form of the function uniquely. It allows the following six building blocks $P_{12}, K_{12}, K_{13}, K_{23}, K_{123}, K_{213}$ and the three-point function is

$$
\left\langle\mathcal{O}_{n}^{J_{1}}\left(z_{1}, V_{1}\right) \mathcal{O}_{m}^{J_{2}}\left(z_{2}, V_{2}\right) \mathcal{Q}^{J_{3}}\left(z_{3}, V_{3}\right)\right\rangle=\sum_{a} C_{n m}^{J_{1} J_{2} J_{3}}(a) P_{12}^{a} K_{12}^{b} K_{13}^{c} K_{123}^{d} K_{23}^{e} K_{213}^{f}
$$

The exponents are related by the charge conservation relations $b+c+d=J_{1}, b+e+f=J_{2}$, $c+d+e+f=J_{3}$ and conformal dimension matching relations $a+b+c+d+2 f=\Delta_{n}^{J_{1}}$, $a+b+e+2 d+f=\Delta_{m}^{J_{2}}$. This yields

$$
\begin{aligned}
b & =\frac{1}{2}\left(J_{1}+J_{2}-J_{3}\right) \\
c & =\frac{1}{2}\left(J_{1}+J_{3}-\Delta_{m}^{J_{2}}+a\right) \\
d & =\frac{1}{2}\left(\Delta_{m}^{J_{2}}-J_{2}-a\right) \\
e & =\frac{1}{2}\left(J_{2}+J_{3}-\Delta_{n}^{J_{1}}+a\right) \\
f & =\frac{1}{2}\left(\Delta_{n}^{J_{1}}-J_{1}-a\right)
\end{aligned}
$$


with one free parameter $a$. For a general combination of $J_{1}, J_{2}, J_{3}$ the form of the three-point function is not fixed by superconformal symmetry. Taking derivatives with respect to $a$ should yield (nilpotent) invariants, each of which gives rise to an independent structure constant $C$. The case that we are going to consider is an extremal correlator with $J_{1}=J_{2}+J_{3}$ where we might hope for another constraint. Assuming that $e$ and $f$ must be positive, the form of this extremal correlator would be uniquely fixed, because $e+f=0$ with the only solution $e=f=0$ and

$$
a=\Delta_{n}^{J_{1}}-J_{1} \quad \text { for } J_{1}=J_{2}+J_{3} .
$$

The single structure constant in the BMN limit could then be read off from the correlator of the singlet operator in [21]

$$
C_{n m}^{J, J r, J(1-r)} \sim \frac{2 g_{2} \sqrt{1-r} \sin ^{2}(\pi n r)}{\sqrt{J r} \pi^{2}\left(n^{2}-\frac{m^{2}}{r^{2}}\right)^{2}} \times \frac{m^{2}}{r^{2}} .
$$

We now set $V_{2}=V_{3}=\bar{V}_{1}$ and perform the variations $\left(\delta_{1} \delta_{2}\right)^{l}$ on (5.34). On the left hand side we get the descendant operators multiplied by their normalisation constants $N_{a, n}^{J,(l / 2)}$ and $N_{b, m}^{J r,(l / 2)}$. On the right hand side we first act with $\delta_{1}^{l}$. This affects only $P_{12}$. It gives rise to $l$ powers of $\Theta_{12}$ which need to be saturated by $\delta_{2}^{l}$ when we set $\Theta=0$. Therefore $\left(\delta_{1} \delta_{2}\right)^{l}$ effectively acts only on $P_{12}$. This results in the same expressions as for the two-point function (5.26) of the operator $\mathcal{O}_{n}^{J}$ and a factor of $\left(N_{a, n}^{J,(l / 2)}\right)^{2}$. The generic descendant correlator for normalised operators is thus

$$
\begin{aligned}
\left\langle\mathcal{O}_{a, n}^{J,(l / 2)}\left(z_{1}, V\right)\right. & \left.\mathcal{O}_{b, m}^{J r,(l / 2)}\left(z_{2}, \bar{V}\right) \mathcal{Q}^{J(1-r)}\left(z_{3}, \bar{V}\right)\right\rangle\left.\right|_{0} \\
= & \frac{\left(N_{a, n}^{J,(l / 2)} / N_{b, m}^{J r,(l / 2)}\right) C_{m n}^{J, J r, J(1-r)} J_{12, a b}}{\left|x_{12}\right|^{\Delta_{n}^{J}+\Delta_{m}^{J r}-J(1-r)+l}\left|x_{23}\right|^{\Delta_{m}^{J r}+J(1-r)-\Delta_{n}^{J}}\left|x_{13}\right|^{\Delta_{n}^{J}+J(1-r)-\Delta_{m}^{J r}}}
\end{aligned}
$$

The normalisation constants $N_{a, n}^{J,(l)}$ can be found in App. D. In the BMN limit and to leading order the quotient of normalisation constants can only be $1, \mathrm{nr} / \mathrm{m}$ or $n^{2} r^{2} / \mathrm{m}^{2}$ which effectively replaces the last factor in (5.37) by $m^{2} / r^{2}, m n / r$ and $n^{2}$, respectively. One should keep in mind that this form of the function is based on some assumptions. In principle, one should analyse all three-point covariants relevant to these operators, a task beyond the scope of this work. It needs to be compared to some explicit calculations. For instance it does coincide with the correlators of antisymmetric and symmetric-traceless BMN operators in [21,22.

Three BMN operators. A three-point function of three BMN operators is less constrained than the above three-point functions and none of these correlators has been calculated so far. We will therefore not investigate it here.

\section{Discussion and Outlook}

In this paper we have investigated the implications of superconformal symmetry on the BMN operators with two charge defects. It was seen how the BMN operators fill out 
multiplets of SU(4) and $\mathrm{SU}(2,2 \mid 4)$ and an abstract way of defining them was found. We have determined the form of the operators with scalar impurities at finite charge $J$ and their anomalous dimension. This was then used to derive the form of all other bosonic operators by supersymmetry. Finally, we have presented superspace three-point functions involving BMN operators which agree with previously computed correlators.

The main result of the group theoretical considerations is that the long supermultiplets of $\mathcal{N}=4$ super Yang-Mills theory whose lowest dimensional operators are scalars of naive dimension $J+2$ transforming in the irreducible representation $[0, J, 0]$ of $\mathrm{SU}(4)$ contain exactly the BMN operators with two impurities. This is an alternative definition of these operators as opposed to the heuristic construction of BMN. The new definition enables one to apply useful results of superconformal symmetry and representation theory of $\mathrm{SU}(2,2 \mid 4)$ to the set of BMN operators.

We have found the exact one-loop, planar form of the BMN operators at finite $J$, see (3.3). The obtained phase factors differ from any of the previously conjectured ones. This is without consequences in the BMN limit because the modification is negligible. It is, however, crucial when $J$ is small. We find that some of the operators with small $J$ coincide with operators which have been intensely studied in recent years. Most importantly this is the Konishi operator at $J=0, n=1$. Furthermore, some of the dimension four operators studied in [15] coincide with our expressions. We are able to reproduce their anomalous dimension with a single expression that remains valid in the BMN limit. Furthermore, it can be seen that the mixing pattern of single-trace and double-trace operators [21 persists at small $J$, see for example 40, 41, 15]. We thus found a class of operators that interpolates between the BMN limit and operators at low dimension.

In fact, it seems to be more useful to classify operators of $\mathcal{N}=4 \mathrm{SYM}$ by their number of defects than by their dimension. The larger the number of defects, the further the operators are 'away' from the protected operators. As the number of impurities increases the number of operators vastly increases and operator mixing becomes more and more complicated. In contrast, increasing the charge of an operator while keeping the number of defects constant adds only a manageable amount of combinatorics to the problem. The additional charged fields act as an inert background to the original operator in many respects.

In this proposed classification a single-trace operator is characterised by several numbers. The most important ones are the number of defects, $k$, and the $\mathrm{SO}(4)$ and $\mathrm{SO}(3,1)$ representations. The total spin of these representations is bounded from above by $k-2$. Furthermore, there is the $\mathrm{SO}(2)$ charge $J$. If the charge is reasonably large compared to the number of defects, the defects can be viewed as a dilute gas and one should expect that everything depends 'smoothly' on $J$. As proposed in [1] the operators with single-charge defects $\left(\phi_{i}, \mathcal{D}_{\mu}, \psi\right)$ will then organise themselves in terms of $k-1$ mode numbers. In such a way the spectrum of strings on a plane-wave is obtained. The operators with multiple-charge defects $\left(\bar{Z}, F_{\mu \nu}, \bar{\psi}, \ldots\right)$, which seemingly do not fit into the string spectrum, were expected [1] to become infinitely massive in the BMN limit and decouple from the low-lying modes. Interestingly, we find that in the case of two defects this does not happen. Nevertheless, if for a different reason, the agreement of spectra is 
not spoiled by the presence of additional insertions. Operators with multiple-charge defects are hidden within the ordinary ones with single-charge defects by operator mixing. They give rise to an additional mode instead of an exceptional operator. Therefore all operators are classified by a single mode number and their correlation functions depend 'smoothly' on it. One might conjecture that this holds in general. We have also seen that the same mode decomposition of operators can be extended all the way down to the smallest possible charge $J$. (Certainly, only low winding numbers would be allowed for these and the term 'mode decomposition' becomes somewhat inappropriate). Again, this might hold in general. Then the remaining characteristic numbers are given by a set of $k-1$ mode numbers. In that case, the spectrum would be very similar to the spectrum of a single string in free string theory. Including multiple-trace operators we would naturally arrive at an interacting string theory. This exhausts the spectrum of local operators in the gauge theory. Consequently, this characterisation might be very appealing for the general AdS/CFT correspondence away from the plane-wave limit.

In this context the BMN limit of an arbitrary operator could be obtained by taking the charge of the operator to infinity while keeping all other classifying numbers fixed. In the case of operators with two defects we have seen that the mode decomposition includes all operators. There are no exceptional operators which become infinitely massive as suggested in [1. It follows that the complete set of operators (with a finite mode number) survives in the BMN limit. Although we have not found any infinitely massive operators in the BMN limit so far, it might well be that they exist among the operators with more than two defects. If so, the classification scheme would have to be enhanced accordingly.

We note that the form of the primary BMN operator (1.3) is only the lowest order approximation to its full form. Redefinitions are required at higher genus and higher loops. We have illustrated the modifications that occur at genus one, higher corrections will be similar. Beyond one-loop one should expect mixing between operators with scalar, fermionic and derivative insertions. The diagonalisation would involve a redefinition of the primary operators $\mathcal{O}_{n}^{J}$ by operators with equal quantum numbers $\left(\mathcal{O}_{k}^{J-2,(2)}, \mathcal{O}_{k}^{J-4,(4)}\right)$. One may argue that this is negligible in the BMN limit: Due to the dilute gas property interactions involving both impurities should be suppressed. This is true in the planar limit, but not in general. The additional pieces in the amplitudes of singlet operators in 21] are exclusively due to interactions between both impurities. We should stress that in this work we have mostly been considering full, diagonalised operators. If the correspondence to strings on plane-waves is true, one should find that their anomalous dimensions agree with the eigenvalues of the string Hamiltonian. Current attempts to compare both theories [19, 27, 28, 29, 30] do not try to accomplish that, however. They aim at comparing matrix elements at the level of bare operators/states.

In order to show that all the BMN operators belong to the same supermultiplet we have worked out supersymmetry descendants of the primary operator, see (4.24). We have also shown how supersymmetry relates the mixing matrices. This reduces the complexity of future calculations, as only the primary operator has to be taken into account. Supersymmetry is then used to derive the corresponding statements for the descendants. The form of the descendants in App. B can be used as a dictionary.

Using superconformal symmetry we have found two-point and three-point functions of 
BMN operators. We have worked out the two-point correlators for descendants operators, see (5.25) and obtained the exact expressions for the normalisation coefficients of the variation of the operators. A complete set of correlators and normalisation constants is found in App. C] and D. Three-point functions of BMN operators were presented, most importantly (5.38). They explain the relations between correlators that have been determined recently [21,22]. In principle, these should enable one to derive expressions for a large class of descendant correlators.

Several questions concerning BMN operators at finite charge and their classification suggest themselves. An extension of the current analysis to operators with more than two defects would be interesting. In particular an enumeration of such operators and their explicit form at one-loop and at the planar level might lead to new insights into the proposed classification. For instance, one might expect some new features to appear at the level of four defects. The representation $[0, J, 0]$ with dimension $J+4$ is not on the unitary bound and its constituents might therefore behave quite differently. For example their anomalous dimensions are not required to be positive. This representation might also lead to the simplest examples of operators that become infinitely massive in the BMN limit, should these exist at all. Furthermore, in the low charge regime we would expect to find the operators with $J=0$ investigated in [48. Alternatively, one could work in the opposite direction and try to generalise some results involving low-dimensional operators, like the Konishi operators, to arbitrary charge and to the BMN limit. A twoloop generalisation of some of the results involving BMN operators at finite $J$ would also be useful. For example, the anomalous dimension depends on two parameters, $J$ and $n$. By investigating the dependence of the two-loop result on the additional parameters one might be able to guess the structure of the higher-loop anomalous dimensions. In that sense the BMN operators and the BMN limit might lead to a better understanding of $\mathcal{N}=4 \mathrm{SYM}$ and the AdS/CFT correspondence in general.

\section{Acknowledgements}

The author would like to thank Gleb Arutyunov, Thomas Klose, Stefano Kovacs, Charlotte Kristjansen, Ari Pankiewicz and Jan Plefka for useful discussions. In particular I am grateful to Matthias Staudacher for many helpful suggestions and comments on the manuscript. 


\section{A Spinors in $D=9+1$}

We use indices the $M, N, \ldots=0, \ldots, 9$ for vectors and indices $A, B, \ldots=1, \ldots, 16$ for spinors. Some $\mathrm{SO}(9,1)$ invariant tensors are the metric $\eta_{M N}=\operatorname{diag}(-,+, \ldots,+)$, the antisymmetric tensor $\varepsilon_{M N O P Q R S T U V}$, and the tensors $\Sigma_{A B}^{M}$ and $\tilde{\Sigma}_{M}^{A B}$ relating two spinor indices with one vector index. The $\Sigma$ matrices (where the spinor indices are commonly suppressed) satisfy the Clifford algebra

$$
\Sigma_{M} \tilde{\Sigma}_{N}+\Sigma_{N} \tilde{\Sigma}_{M}=2 \eta_{M N}
$$

\section{Normal ordering}

$$
\begin{aligned}
\Sigma_{M} \tilde{\Sigma}_{N}= & \Sigma_{M N}+\eta_{M N} \\
\Sigma_{M} \tilde{\Sigma}_{N} \Sigma_{R}= & \Sigma_{M N R}+\eta_{M N} \Sigma_{R}-\eta_{M R} \Sigma_{N}+\eta_{N R} \Sigma_{M}, \\
\Sigma_{M} \tilde{\Sigma}_{N} \Sigma_{R} \tilde{\Sigma}_{S}= & \Sigma_{M N R S} \\
& +\eta_{M N} \Sigma_{R S}-\eta_{M R} \Sigma_{N S}+\eta_{M S} \Sigma_{N R} \\
& +\eta_{N R} \Sigma_{M S}-\eta_{N S} \Sigma_{M R}+\eta_{R S} \Sigma_{M N} \\
& +\eta_{M N} \eta_{R S}-\eta_{M R} \eta_{N S}+\eta_{M S} \eta_{N R}
\end{aligned}
$$

where $\Sigma_{M N R \ldots}$ is defined to be the antisymmetrised ('normal ordered') product of $\Sigma \mathrm{s}$

$$
\Sigma_{M N R \ldots}=\Sigma_{[M} \tilde{\Sigma}_{N} \Sigma_{R} \cdots
$$

and tilded symbols are obtained by all $\Sigma$ s replaced by $\tilde{\Sigma}$ s and vice versa.

Chisholm identities (for a Clifford algebra of $d \Sigma_{i}$ matrices)

$$
\begin{aligned}
\Sigma_{i} \Sigma_{(n)} \Sigma^{i} & =(-1)^{n}(d-2 n) \Sigma_{(n)}, \\
\Sigma_{i j} \Sigma_{(n)} \Sigma^{i j} & =\left(d-(d-2 n)^{2}\right) \Sigma_{(n)}, \\
\sum_{i j k} \Sigma_{(n)} \Sigma^{i j k} & =(-1)^{n}(d-2 n)\left(3 d-2-(d-2 n)^{2}\right) \Sigma_{(n)},
\end{aligned}
$$

We have suppressed the tilde on every second $\Sigma$ and $\Sigma_{(n)}$ denotes an normal ordered product of $n \Sigma_{i}$ s. This rule is also applicable when a subset of $d$ of the $D=9+1 \Sigma$ s is considered.

\section{Symmetries}

$$
\begin{aligned}
\Sigma_{M}^{\top} & =+\Sigma_{M}=\Sigma_{M}^{*}=\Sigma_{M}^{\dagger}, \\
\Sigma_{M N}^{\mathrm{\top}} & =-\tilde{\Sigma}_{M N}, \\
\Sigma_{M N P}^{\mathrm{T}} & =-\Sigma_{M N P}, \\
\Sigma_{M N P Q}^{\mathrm{T}} & =+\tilde{\Sigma}_{M N P Q},
\end{aligned}
$$


Dualisations (opposite signs for $\left.\tilde{\Sigma}_{\mathrm{s}}\right)$

$$
\begin{aligned}
1= & +\frac{1}{10 !} \varepsilon_{M N O P Q R S T U V} \Sigma^{M N O P Q R S T U V} \\
\Sigma_{M}= & -\frac{1}{9 !} \varepsilon_{M N O P Q R S T U V} \Sigma^{N O P Q R S T U V} \\
\Sigma_{M N}= & -\frac{1}{8 !} \varepsilon_{M N O P Q R S T U V} \Sigma^{O P Q R S T U V} \\
\Sigma_{M N O}= & +\frac{1}{7 !} \varepsilon_{M N O P Q R S T U V} \Sigma^{P Q R S T U V} \\
& \ldots
\end{aligned}
$$

\section{Fierz identities}

$$
\begin{aligned}
a^{[A} b^{B]} & =\frac{1}{16 \cdot 3 !}\left(a^{\top} \Sigma^{M N P} b\right) \tilde{\Sigma}_{M N P}^{A B} \\
a_{[A} b_{B]} & =\frac{1}{16 \cdot 3 !}\left(a^{\top} \tilde{\Sigma}^{M N P} b\right) \Sigma_{A B}^{M N P} \\
a^{\{A} b^{B\}} & =\frac{1}{16}\left(a^{\top} \Sigma^{M} b\right) \tilde{\Sigma}_{M}^{A B}+\frac{1}{16 \cdot 2 \cdot 5 !}\left(a^{\top} \Sigma_{M N O P Q} b\right) \tilde{\Sigma}_{M N O P Q}^{A B} \\
a_{\{A} b_{B\}} & =\frac{1}{16}\left(a^{\top} \tilde{\Sigma}_{M} b\right) \Sigma_{A B}^{M}+\frac{1}{16 \cdot 2 \cdot 5 !}\left(a^{\top} \tilde{\Sigma}_{M N O P Q} b\right) \Sigma_{A B}^{M N O P Q} \\
a^{A} b_{B} & =\frac{1}{16}\left(a^{\top} b\right) \delta_{B}^{A}+\frac{1}{16 \cdot 2 !}\left(a^{\top} \Sigma^{M N} b\right) \tilde{\Sigma}_{M N}^{A}{ }_{B}+\frac{1}{16 \cdot 4 !}\left(a^{\top} \Sigma^{M N P Q} b\right) \tilde{\Sigma}_{M N P Q}{ }_{B}
\end{aligned}
$$

Reduction to $\boldsymbol{D}=\mathbf{3}+\mathbf{1}, \mathcal{N}=\mathbf{4}$. To be able to work with this notation in $\mathcal{N}=4$ SYM we need to split up the vectors in 4 and 6 components. The spacetime vectors are labelled by indices $\mu, \nu, \ldots=0, \ldots, 3$ and the internal vectors by $m, n, \ldots=1, \ldots 6$. Due to this $\mathrm{SO}(3,1) \times \mathrm{SO}(6)$ split we obtain two antisymmetric invariant tensors $\varepsilon_{\mu \nu \rho \sigma}$ and $\varepsilon_{\text {mnpqrs }}$ and one can build an invariant combination of $\Sigma$ s

$$
\begin{aligned}
& \Sigma_{(4)}=\frac{1}{4 !} \varepsilon_{\mu \nu \rho \sigma} \Sigma^{\mu \nu \rho \sigma}=-\frac{1}{6 !} \varepsilon_{m n o p q r} \Sigma^{m n o p q r}=-\Sigma_{(6)} \\
& \tilde{\Sigma}_{(4)}=\frac{1}{4 !} \varepsilon_{\mu \nu \rho \sigma} \tilde{\Sigma}^{\mu \nu \rho \sigma}=+\frac{1}{6 !} \varepsilon_{m n o p q r} \tilde{\Sigma}^{\text {mnopqr }}=+\tilde{\Sigma}_{(6)}
\end{aligned}
$$

with the properties

$$
\Sigma_{(4)}^{2}=\Sigma_{(6)}^{2}=\tilde{\Sigma}_{(4)}^{2}=\tilde{\Sigma}_{(6)}^{2}=-1, \quad \Sigma_{(4)}^{\top}=\tilde{\Sigma}_{(4)}, \quad \Sigma_{(6)}^{\top}=-\tilde{\Sigma}_{(6)} .
$$

These give rise to chiral projectors

$$
P=\frac{1}{2}+\frac{i}{2} \Sigma_{(4)}, \quad \bar{P}=\frac{1}{2}-\frac{i}{2} \Sigma_{(4)} .
$$

Useful identities for the computation of traces involving the projectors are

$$
P \Sigma_{\mu \nu \rho \sigma}=i \varepsilon_{\mu \nu \rho \sigma} P, \quad P \Sigma_{m n o p q r}=i \varepsilon_{m n o p q r} P .
$$

Harmonic coordinates. We introduce a complex internal vector $V_{m}$ with the properties $V^{2}=0,|V|^{2}=1$. Two components of an internal vector are specialised by this, $a_{+}=a \cdot V, a_{-}=a \cdot \bar{V}$. The remaining four are labelled by indices $i, j, \ldots=1, \ldots, 4$. There are two projectors

$$
P_{+}=\frac{1}{2} \Sigma_{-} \tilde{\Sigma}_{+}, \quad P_{-}=\frac{1}{2} \Sigma_{+} \tilde{\Sigma}_{-}
$$

which effectively project to spinors of the $\mathrm{SO}(7,1)$ subgroup of $\mathrm{SO}(9,1)$ that leaves $V$ and $\bar{V}$ invariant. 


\section{B Descendant operators}

In this appendix we present all bosonic BMN operators with two defects and how they are related by supersymmetry transformations that do not change the number of defects.

\section{Definitions}

$$
\begin{aligned}
\delta & =\epsilon^{\top} \tilde{\Sigma}_{+} D \\
e_{M N} & =\epsilon^{\top} \tilde{\Sigma}_{+M N} \epsilon \\
\kappa_{n}^{J} & =\sqrt{8} \sqrt{\frac{g_{\mathrm{YM}}^{2} N}{8 \pi^{2}}} \sin \frac{\pi n}{J+3} \sim \sqrt{\lambda^{\prime}} n \\
e_{M+} & =e_{M-}=0
\end{aligned}
$$

Fierz identities

level 2

$$
\tilde{\Sigma}_{+} \epsilon \epsilon^{\top} \tilde{\Sigma}_{+}=\frac{1}{16} e_{M N} \tilde{\Sigma}_{+} \Sigma^{M N}
$$

level 4

$$
\begin{aligned}
e^{i j} e^{k l} & =e^{[i j} e^{k l]}+e^{[k}{ }_{\mu} \delta^{l][j} e^{i] \mu}+\frac{1}{6} \delta^{i[k} \delta^{l] j} e^{m n} e_{m n}, \\
e^{i j} e^{k \mu} & =e^{[i j} e^{k] \mu}+\frac{2}{3} \delta^{k[i} e^{j] \nu} e^{\mu}{ }_{\nu} \\
e^{i \mu} e^{j \nu} & =-e^{i j} e^{\mu \nu}+\frac{1}{4} \eta^{\mu \nu} e^{i \rho} e^{j}{ }_{\rho}+\frac{1}{4} \delta^{i j} e^{\mu k} e^{\nu}{ }_{k}, \\
e^{i k} e^{j} & =\frac{1}{2} e^{i \mu} e^{j}{ }_{\mu}+\frac{1}{4} \delta^{i j} e^{l k} e_{l k}, \\
e^{i k} e^{\mu}{ }_{k} & =e^{i \rho} e^{\mu}{ }_{\rho} \\
e^{i \mu} e_{i \mu} & =0 \\
e^{i j} e_{i j} & =-e^{\mu \nu} e_{\mu \nu} \\
e_{[M N} e_{O P]} & =-\frac{1}{4 !} \varepsilon_{+-M N O P Q R S T} e^{[Q R} e^{S T]}
\end{aligned}
$$

level 6

$$
\begin{aligned}
e^{i \mu} e_{k \mu} e^{k j} & =e^{i j} e^{k l} e_{k l} \\
e^{i k} e_{l k} e^{l j} & =\frac{3}{4} e^{i j} e^{k l} e_{k l}
\end{aligned}
$$

level 8

$$
e^{i \mu} e_{j \mu} e^{j \nu} e_{i \nu}=2 e^{i j} e_{i j} e^{k l} e_{k l}
$$




\section{Variations of $\mathcal{O}_{n}^{J}$}

$$
\begin{aligned}
\delta^{2} \mathcal{O}_{n}^{J}= & \sqrt{2} e^{i j} \kappa_{n}^{J} \mathcal{O}_{i j, n}^{J,(1)}+2 \sqrt{2} e^{\mu \nu} \mathcal{O}_{\mu \nu, n}^{J,(1)}+2 \sqrt{2} e^{\mu i} \mathcal{O}_{\mu i, n}^{J,(1)} \\
\delta^{4} \mathcal{O}_{n}^{J}= & -6\left(\kappa_{n}^{J}\right)^{2} e^{i \mu} e^{j}{ }_{\mu} \mathcal{O}_{(i j), n}^{J,(2)}+24 e^{\mu i} e^{\nu}{ }_{i} \mathcal{O}_{(\mu \nu), n}^{J,(2)}+16 \sqrt{2} \kappa_{n}^{J} e^{\mu j} e^{i}{ }_{j} \mathcal{O}_{\mu i, n}^{J,(2)} \\
& +4 \sqrt{2} \kappa_{n}^{J} e^{i j} e_{i j} \mathcal{O}_{n}^{J,(2)}+4 \sqrt{6} \kappa_{n}^{J} e^{[M N} e^{R S]} \mathcal{O}_{[M N R S]}^{J,(2)} \\
& -2 e^{j k} e_{j k} \frac{N_{0}^{-J-2}}{\sqrt{J+3}} \cos \frac{\pi n}{J+3} \operatorname{Tr}\left(\mathcal{D}^{\mu} \mathcal{D}_{\mu} Z+\left[i \phi^{i},\left[i \phi_{i}, Z\right]\right]+i \psi^{\top} \Sigma_{+} \psi\right) Z^{J+1} \\
\delta^{6} \mathcal{O}_{n}^{J}= & 120 \sqrt{2}\left(\kappa_{n}^{J}\right)^{2} e^{i j} e^{k l} e_{k l} \mathcal{O}_{[i j], n}^{J,(3)}+240 \sqrt{2} \kappa_{n}^{J} e^{\mu \nu} e^{\rho \sigma} e^{\rho \sigma} \mathcal{O}_{[\mu \nu], n}^{J,(3)} \\
& -160 \sqrt{2}\left(\kappa_{n}^{J}\right)^{2} e^{\mu j} e_{\nu j} e^{\nu i} \mathcal{O}_{\mu i, n}^{J,(3)}+\mathrm{EOM} \\
\delta^{8} \mathcal{O}_{n}^{J}= & -2240\left(\kappa_{n}^{J}\right)^{2} e^{i j} e_{i j} e^{k l} e_{k l} \mathcal{O}_{n}^{J,(4)}+\mathrm{EOM}
\end{aligned}
$$

\section{Variations of $\mathcal{O}_{n}^{J,(2)}$}

$$
\begin{aligned}
& \delta^{2} \mathcal{O}_{n}^{J,(2)}=e^{i j} \kappa_{n}^{J} \mathcal{O}_{[i j], n}^{J,(3)}-2 e^{\mu \nu} \mathcal{O}_{[\mu \nu], n}^{J,(3)}, \\
& \delta^{4} \mathcal{O}_{n}^{J,(2)}=-4 \sqrt{2} \kappa_{n}^{J} e^{i j} e_{i j} \mathcal{O}_{n}^{J,(4)}
\end{aligned}
$$

\section{Descendant Operators}

level 0

$$
\begin{aligned}
\mathcal{O}_{n}^{J}=\frac{N_{0}^{-J-2}}{\sqrt{J+3}}\left[\frac{1}{2} \sum_{p=0}^{J}\right. & \cos \frac{\pi n(2 p+3)}{J+3} \operatorname{Tr} \phi_{i} Z^{p} \phi_{i} Z^{J-p} \\
& \left.-2 \cos \frac{\pi n}{J+3} \operatorname{Tr} \bar{Z} Z^{J+1}\right]
\end{aligned}
$$

level 2

$$
\begin{aligned}
& \mathcal{O}_{[i j], n}^{J,(1)}=\frac{N_{0}^{-J-3}}{\sqrt{J+3}} \sum_{p=0}^{J+1} i \sin \frac{\pi n(2 p+2)}{J+3} \operatorname{Tr} \phi_{[i} Z^{p} \phi_{j]} Z^{J+1-p} \\
& \mathcal{O}_{[\mu \nu], n}^{J,(1)}=\frac{N_{0}^{-J-2}}{\sqrt{J+3}}\left[\frac{1}{8 \sqrt{2}} \sum_{p=0}^{J} \cos \frac{\pi n(2 p+3)}{J+3} \operatorname{Tr} \psi^{\top} Z^{p} \Sigma_{+\mu \nu} \psi Z^{J-p}\right. \\
& \left.+\frac{1}{\sqrt{2}} \cos \frac{\pi n}{J+3} \operatorname{Tr} F_{\mu \nu} Z^{J+1}\right] \\
& \mathcal{O}_{\mu i, n}^{J,(1)}=\frac{N_{0}^{-J-2}}{\sqrt{J+3}}\left[\frac{1}{\sqrt{2}} \sum_{p=0}^{J} \cos \frac{\pi n(2 p+3)}{J+3} \operatorname{Tr} \phi_{i} Z^{p} \mathcal{D}_{\mu} Z Z^{J-p}\right. \\
& +\sqrt{2} \cos \frac{\pi n}{J+3} \operatorname{Tr} \mathcal{D}_{\mu} \phi_{i} Z^{J+1} \\
& \left.+\frac{1}{8 \sqrt{2}} \sum_{p=0}^{J} \cos \frac{\pi n(2 p+3)}{J+3} \operatorname{Tr} \psi^{\top} Z^{p} \Sigma_{+\mu i} \psi Z^{J-p}\right]
\end{aligned}
$$


level 4

$$
\begin{aligned}
\mathcal{O}_{(i j), n}^{J,(2)}= & \frac{N_{0}^{-J-4}}{\sqrt{J+3}} \sum_{p=0}^{J+2} \cos \frac{\pi n(2 p+1)}{J+3} \operatorname{Tr} \phi_{(i} Z^{p} \phi_{j} Z^{J+2-p} \\
\mathcal{O}_{(\mu \nu), n}^{J,(2)}= & \frac{N_{0}^{-J-2}}{\sqrt{J+3}}\left[\frac{1}{2} \sum_{p=0}^{J} \cos \frac{\pi n(2 p+3)}{J+3} \operatorname{Tr} \mathcal{D}_{(\mu} Z Z^{p} \mathcal{D}_{\nu)} Z Z^{J-p}\right. \\
& \left.+\frac{1}{2} \cos \frac{\pi n}{J+3} \operatorname{Tr} \mathcal{D}_{(\mu} \mathcal{D}_{\nu)} Z Z^{J+1}\right] \\
\mathcal{O}_{\mu i, n}^{J,(2)}= & \frac{N_{0}^{-J-3}}{\sqrt{J+3}} \sum_{p=0}^{J+1} i \sin \frac{\pi n(2 p+2)}{J+3} \operatorname{Tr} \phi_{i} Z^{p} \mathcal{D}_{\mu} Z Z^{J+1-p} \\
\mathcal{O}_{n}^{J,(2)}= & \frac{N_{0}^{-J-3}}{\sqrt{J+3}}\left[\frac{1}{8} \sum_{p=0}^{J+1} i \sin \frac{\pi n(2 p+2)}{J+3} \operatorname{Tr} \psi^{\top} Z^{p} \Sigma_{+} \psi Z^{J+1-p}\right. \\
& \left.\quad+\frac{1}{2} \sum_{p=0}^{J+1} i \sin \frac{\pi n(2 p+2)}{J+3} \operatorname{Tr} \phi_{i} Z^{p}\left[i \phi_{i}, Z\right] Z^{J+1-p}\right] \\
\mathcal{O}_{[M N R S], n}^{J,(2)}= & \frac{N_{0}^{-J-3}}{32 \sqrt{3} \sqrt{J+3}} \sum_{p=0}^{J+1} i \sin \frac{\pi n(2 p+2)}{J+3} \operatorname{Tr} \psi^{\top} Z^{p} \Sigma_{+M N R S} \psi Z^{J+1-p}
\end{aligned}
$$

level 6

$$
\begin{aligned}
\mathcal{O}_{[\mu \nu], n}^{J,(3)}=\frac{N_{0}^{-J-3}}{\sqrt{J+3}}[ & \frac{1}{2} \sum_{p=0}^{J+1} i \sin \frac{\pi n(2 p+2)}{J+3} \operatorname{Tr} \mathcal{D}_{[\mu} Z Z^{p} \mathcal{D}_{\nu]} Z Z^{J+1-p} \\
& \left.-\frac{1}{16} \sum_{p=0}^{J+1} i \sin \frac{\pi n(2 p+2)}{J+3} \operatorname{Tr} \psi^{\top} \Sigma_{+\mu \nu} Z^{p}[i \psi, Z] Z^{J+1-p}\right] \\
\mathcal{O}_{[i j], n}^{J,(3)}=\frac{N_{0}^{-J-4}}{\sqrt{J+3}}[ & \frac{1}{8 \sqrt{2}} \sum_{p=0}^{J+2} \cos \frac{\pi n(2 p+1)}{J+3} \operatorname{Tr} \psi^{\top} \Sigma_{+i j} Z^{p} \psi Z^{J+2-p} \\
& \left.+\frac{1}{\sqrt{2}} \sum_{p=0}^{J+2} \cos \frac{\pi n(2 p+1)}{J+3} \operatorname{Tr} \phi_{i} Z^{p}\left[i \phi_{j}, Z\right] Z^{J+2-p}\right] \\
\mathcal{O}_{\mu i}^{J,(3)}=\frac{N_{0}^{-J-4}}{\sqrt{J+3}}[ & \frac{1}{\sqrt{2}} \sum_{p=0}^{J+2} \cos \frac{\pi n(2 p+1)}{J+3} \operatorname{Tr} \phi_{i} Z^{p} \mathcal{D}_{\mu} Z Z^{J+2-p} \\
& \left.-\frac{1}{8 \sqrt{2}} \sum_{p=0}^{J+2} \cos \frac{\pi n(2 p+1)}{J+3} \operatorname{Tr} \psi^{\top} \Sigma_{+\mu i} Z^{p} \psi Z^{J+2-p}\right]
\end{aligned}
$$


level 8

$$
\begin{aligned}
\mathcal{O}_{n}^{J,(4)}=\frac{N_{0}^{-J-4}}{\sqrt{J+3}}[ & \frac{1}{4} \sum_{p=0}^{J+2} \cos \frac{\pi n(2 p+1)}{J+3} \operatorname{Tr} \mathcal{D}^{\mu} Z Z^{p} \mathcal{D}_{\mu} Z Z^{J+2-p} \\
& -\frac{1}{8} \sum_{p=0}^{J+2} \cos \frac{\pi n(2 p+1)}{J+3} \operatorname{Tr} \psi^{\top} \Sigma_{+} Z^{p}[i \psi, Z] Z^{J+2-p} \\
& \left.+\frac{1}{4} \sum_{p=0}^{J+2} \cos \frac{\pi n(2 p+1)}{J+3} \operatorname{Tr}\left[i \phi_{i}, Z\right] Z^{p}\left[i \phi_{i}, Z\right] Z^{J+2-p}\right]
\end{aligned}
$$

\section{Variations of the scalar superspace propagator}

In this appendix we present all components of the scalar superspace propagator corresponding to bosonic BMN operators with two defects.

\section{Definitions}

$$
\begin{aligned}
P_{12} & =\frac{1}{\left|y_{12}\right|^{\Delta}\left|\bar{y}_{12}\right|^{\Delta}} \\
J_{12, \mu \nu} & =\eta_{\mu \nu}-2 \frac{x_{12, \mu} x_{12, \nu}}{x_{12}^{2}}, \\
J_{12, m n} & =\delta_{m n} . \\
y_{12}^{\mu} & =x_{12}^{\mu}+\frac{i}{2} \Theta_{12}^{\top} \Sigma_{(4)} \Sigma^{\mu} \Theta_{12}, \\
e_{12}^{\mu} & =-2 \epsilon_{1}^{\top} \tilde{\Sigma}_{+} P \Sigma^{\mu} \tilde{\Sigma}_{-} \epsilon_{2} .
\end{aligned}
$$

\section{Variations of half a scalar propagator}

$$
\begin{aligned}
\delta \frac{1}{y_{12}^{\Delta}} & =-\Delta \frac{y_{12} \cdot e_{12}}{\left|y_{12}\right|^{\Delta+2}} \\
\delta^{2} \frac{1}{y_{12}^{\Delta}} & =\Delta \frac{(\Delta+2)\left(y_{12} \cdot e_{12}\right)^{2}-y_{12}^{2} e_{12}^{2}}{\left|y_{12}\right|^{\Delta+4}} \\
\delta^{3} \frac{1}{y_{12}^{\Delta}} & =-\Delta(\Delta+2) \frac{\left(y_{12} \cdot e_{12}\right)\left((\Delta+4)\left(y_{12} \cdot e_{12}\right)^{2}-3 y_{12}^{2} e_{12}^{2}\right)}{\left|y_{12}\right|^{\Delta+6}} \\
\delta^{4} \frac{1}{y_{12}^{\Delta}} & =\Delta(\Delta+2) \frac{(\Delta+6)(\Delta+4)\left(y_{12} \cdot e_{12}\right)^{4}-6(\Delta+4) y_{12}^{2} e_{12}^{2}\left(y_{12} \cdot e_{12}\right)^{2}+3 y_{12}^{4} e_{12}^{4}}{\left|y_{12}\right|^{\Delta+8}}
\end{aligned}
$$

Fierz identities

$$
\begin{aligned}
\left.\left(y_{12} \cdot e_{12}\right)^{2}\right|_{0}= & \frac{1}{4} x_{12}^{2} \delta_{i k} \delta_{j l} e_{1}^{i j} e_{2}^{k l}-\frac{i}{8} x_{12}^{2} \varepsilon_{+-i j k l} e_{1}^{i j} e_{2}^{k l} \\
& +\frac{1}{4} x_{12}^{2} J_{12, \mu \rho} J_{12, \nu \sigma} e_{1}^{\mu \nu} e_{2}^{\rho \sigma}-\frac{i}{8} x_{12}^{2} J_{12, \mu \kappa} J_{12, \nu \lambda} \varepsilon_{\rho \sigma}^{\kappa \lambda} e_{1}^{\mu \nu} e_{2}^{\rho \sigma} \\
\left.e_{12}^{2}\right|_{0}= & \delta_{i k} \delta_{j l} e_{1}^{i j} e_{2}^{k l}-\frac{i}{2} \varepsilon_{+-i j k l} e_{1}^{i j} e_{2}^{k l} \\
\left.\left(e_{12} \cdot y_{12}\right)\left(\bar{e}_{12} \cdot \bar{y}_{12}\right)\right|_{0}= & \frac{1}{2} x_{12}^{2} J_{12, \mu \nu} \delta_{i j} e_{1}^{\mu i} e_{2}^{\nu j}
\end{aligned}
$$




\section{Variations of the propagator}

level 0

$$
\left.P_{12}^{\Delta}\right|_{0}=\frac{1}{\left|x_{12}\right|^{2 \Delta}}
$$

level 2

$$
\begin{aligned}
\left.\left(\delta_{1} \delta_{2}\right)^{2} P_{12}^{\Delta}\right|_{0}= & \Delta(\Delta-2) e_{1}^{i j} e_{2}^{k l} \frac{\delta_{i[k} \delta_{l] j}}{\left|x_{12}\right|^{2 \Delta+2}} \\
& +\Delta(\Delta+2) e_{1}^{\mu \nu} e_{2}^{\rho \sigma} \frac{J_{12, \mu[\rho} J_{21, \sigma] \nu}}{\left|x_{12}\right|^{2 \Delta+2}} \\
& +2 \Delta^{2} e_{1}^{\mu i} e_{2}^{\nu j} \frac{J_{12, \mu \nu} \delta_{i j}}{\left|x_{12}\right|^{2 \Delta+2}}
\end{aligned}
$$

level 4

$$
\begin{aligned}
\left.\left(\delta_{1} \delta_{2}\right)^{4} P_{12}^{\Delta}\right|_{0}= & 9 \Delta^{2}(\Delta-2)^{2}\left(e_{1}^{i \mu} e_{1 \mu}^{j}\right)\left(e_{2}^{k \nu} e_{2 \nu}^{l}\right) \frac{\delta_{i(k} \delta_{l) l}}{\left|x_{12}\right|^{2 \Delta+4}} \\
& +9 \Delta^{2}(\Delta+2)^{2}\left(e_{1}^{\mu i} e_{1 i}^{\nu}\right)\left(e_{2}^{\rho j} e_{2 j}^{\sigma}\right) \frac{J_{12, \mu(\rho} J_{21, \sigma) \nu}}{\left|x_{12}\right|^{2 \Delta+4}} \\
& +32 \Delta^{2}(\Delta+2)(\Delta-2)\left(e_{1}^{i k} e_{1 k}^{\mu}\right)\left(e_{2}^{\nu l} e_{2 l}^{j}\right) \frac{J_{12, \mu \nu} \delta_{i j}}{\left|x_{12}\right|^{2 \Delta+4}} \\
& +2 \Delta^{2}(\Delta+2)(\Delta-2)\left(e_{1}^{i j} e_{1 i j}\right)\left(e_{2}^{k l} e_{2 k l}\right) \frac{1}{\left|x_{12}\right|^{2 \Delta+4}}, \\
& +6 \Delta^{2}(\Delta+2)(\Delta-2)\left(e_{1}^{[M N} e_{1}^{O P]}\right)\left(e_{2}^{[Q R} e_{2}^{S T]}\right) \frac{J_{12, M Q} J_{12, N R} J_{12, O S} J_{12, P T}}{\left|x_{12}\right|^{2 \Delta+4}}
\end{aligned}
$$

level 6

$$
\begin{aligned}
\left.\left(\delta_{1} \delta_{2}\right)^{6} P_{12}^{\Delta}\right|_{0}= & 900 \Delta^{3}(\Delta+2)(\Delta-2)^{2}\left(e_{1}^{i j} e_{1}^{m n} e_{1 m n}\right)\left(e_{2}^{i j} e_{2}^{r s} e_{2 r s}\right) \frac{\delta_{i[k} \delta_{l] j}}{\left|x_{12}\right|^{2 \Delta+6}} \\
& +900 \Delta^{3}(\Delta+2)^{2}(\Delta-2)\left(e_{1}^{\mu \nu} e_{1}^{\kappa \lambda} e_{1 \kappa \lambda}\right)\left(e_{2}^{\rho \sigma} e_{2}^{\tau \pi} e_{2 \tau \pi}\right) \frac{J_{12, \mu[\rho} J_{21, \sigma] \nu}}{\left|x_{12}\right|^{2 \Delta+6}} \\
& +800 \Delta^{2}(\Delta+2)^{2}(\Delta-2)^{2}\left(e_{1}^{\mu k} e_{1 \rho k} e_{1}^{\rho i}\right)\left(e_{2}^{\nu l} e_{2 \sigma l} e_{2}^{\sigma j}\right) \frac{J_{12, \mu \nu} \delta_{i j}}{\left|x_{12}\right|^{2 \Delta+6}}
\end{aligned}
$$

level 8

$$
\left.\left(\delta_{1} \delta_{2}\right)^{8} P_{12}^{\Delta}\right|_{0}=19600 \Delta^{4}(\Delta+2)^{2}(\Delta-2)^{2}\left(e_{1}^{i j} e_{1 i j} e_{1}^{k l} e_{1 k l}\right)\left(e_{1}^{m n} e_{1 m n} e_{1}^{r s} e_{1 r s}\right) \frac{1}{\left|x_{12}\right|^{2 \Delta+8}}
$$

\section{Normalisation coefficients}

We present the normalisation coefficients of the supersymmetry variations of BMN operators. The variations can be written in a general form as

$$
\delta^{l} \mathcal{O}_{n}^{J}=\sum_{a} N_{a, n}^{J,(l / 2)}\left(\epsilon^{l}\right)^{a} \mathcal{O}_{a, n}^{J(l / 2)} .
$$


The variation parameters $\left(\epsilon^{l}\right)^{a}$ are those from the previous appendices and the operators are canonically normalised. The the right hand side of the following table can be read off from App. C. The left hand side is from App. B. it is the one-loop planar approximation of the full result. The anomalous dimension is $\delta \Delta_{n}^{J}=\Delta_{n}^{J}-J-2$.

$$
\begin{aligned}
& 1=N_{n}^{J}=1, \\
& \sqrt{2} \kappa_{n}^{J} \approx N_{[i j], n}^{J,(1)}=\left(\delta \Delta_{n}^{J}\right)^{1 / 2}\left(2+\delta \Delta_{n}^{J}\right)^{1 / 2}, \\
& 2 \sqrt{2} \approx N_{[\mu \nu], n}^{J,(1)}=\left(2+\delta \Delta_{n}^{J}\right)^{1 / 2}\left(4+\delta \Delta_{n}^{J}\right)^{1 / 2}, \\
& 2 \sqrt{2} \approx N_{\mu i, n}^{J,(1)}=\sqrt{2}\left(2+\delta \Delta_{n}^{J}\right), \\
& -6\left(\kappa_{n}^{J}\right)^{2} \approx N_{(i j), n}^{J,(2)}=-3 \delta \Delta_{n}^{J}\left(2+\delta \Delta_{n}^{J}\right), \\
& 24 \approx N_{(\mu \nu), n}^{J,(2)}=3\left(2+\delta \Delta_{n}^{J}\right)\left(4+\delta \Delta_{n}^{J}\right), \\
& 16 \sqrt{2} \kappa_{n}^{J} \approx N_{\mu i, n}^{J,(2)}=4 \sqrt{2}\left(\delta \Delta_{n}^{J}\right)^{1 / 2}\left(2+\delta \Delta_{n}^{J}\right)\left(4+\delta \Delta_{n}^{J}\right)^{1 / 2}, \\
& 4 \sqrt{2} \kappa_{n}^{J} \approx N_{n}^{J,(2)}=\sqrt{2}\left(\delta \Delta_{n}^{J}\right)^{1 / 2}\left(2+\delta \Delta_{n}^{J}\right)\left(4+\delta \Delta_{n}^{J}\right)^{1 / 2}, \\
& 4 \sqrt{6} \kappa_{n}^{J} \approx N_{[M N R S], n}^{J,(2)}=\sqrt{6}\left(\delta \Delta_{n}^{J}\right)^{1 / 2}\left(2+\delta \Delta_{n}^{J}\right)\left(4+\delta \Delta_{n}^{J}\right)^{1 / 2}, \\
& 120 \sqrt{2}\left(\kappa_{n}^{J}\right)^{2} \approx N_{[i j], n}^{J,(3)}=30\left(\delta \Delta_{n}^{J}\right)\left(2+\delta \Delta_{n}^{J}\right)^{3 / 2}\left(4+\delta \Delta_{n}^{J}\right)^{1 / 2} \text {, } \\
& 240 \sqrt{2} \kappa_{n}^{J} \approx N_{[\mu \nu], n}^{J,(3)}=30\left(\delta \Delta_{n}^{J}\right)^{1 / 2}\left(2+\delta \Delta_{n}^{J}\right)^{3 / 2}\left(4+\delta \Delta_{n}^{J}\right), \\
& -160 \sqrt{2}\left(\kappa_{n}^{J}\right)^{2} \approx N_{\mu i, n}^{J,(3)}=-20 \sqrt{2}\left(\delta \Delta_{n}^{J}\right)\left(2+\delta \Delta_{n}^{J}\right)\left(4+\delta \Delta_{n}^{J}\right), \\
& -2240\left(\kappa_{n}^{J}\right)^{2} \approx N_{n}^{J,(4)}=-140\left(\delta \Delta_{n}^{J}\right)\left(2+\delta \Delta_{n}^{J}\right)^{2}\left(4+\delta \Delta_{n}^{J}\right) .
\end{aligned}
$$

\section{References}

[1] D. Berenstein, J. M. Maldacena and H. Nastase, "Strings in flat space and pp waves from $\mathcal{N}=4$ Super Yang Mills", JHEP 04 (2002) 013, hep-th/0202021.

[2] M. Blau, J. Figueroa-O'Farrill, C. Hull and G. Papadopoulos, "Penrose limits and maximal supersymmetry", Class. Quant. Grav. 19 (2002) L87, hep-th/0201081

[3] R. R. Metsaev, "Type IIB Green-Schwarz superstring in plane wave Ramond-Ramond background", Nucl. Phys. B625 (2002) 70, hep-th/0112044.

[4] M. Spradlin and A. Volovich, "Superstring interactions in a pp-wave background", Phys. Rev. D66 (2002) 086004, hep-th/0204146.

[5] R. Gopakumar, "String interactions in PP-waves", Phys. Rev. Lett. 89 (2002) 171601, hep-th/0205174.

[6] P. Lee, S. Moriyama and J. Park, "Cubic interactions in pp-wave light cone string field theory", Phys. Rev. D66 (2002) 085021, hep-th/0206065

[7] M. Spradlin and A. Volovich, "Superstring interactions in a pp-wave background. II", JHEP 01 (2003) 036, hep-th/0206073.

[8] J. H. Schwarz, "Comments on superstring interactions in a plane-wave background", JHEP 09 (2002) 058, hep-th/0208179. 
[9] A. Pankiewicz, "More comments on superstring interactions in the pp-wave background", JHEP 09 (2002) 056, hep-th/0208209.

[10] A. Pankiewicz and B. Stefanski, Jr., "PP-Wave Light-Cone Superstring Field Theory", hep-th/0210246.

[11] C. Kristjansen, J. Plefka, G. W. Semenoff and M. Staudacher, "A new double-scaling limit of $\mathcal{N}=4$ super Yang-Mills theory and PP-wave strings",

Nucl. Phys. B643 (2002) 3, hep-th/0205033.

[12] D. J. Gross, A. Mikhailov and R. Roiban, "Operators with large $R$ charge in $\mathcal{N}=4$ Yang-Mills theory", Annals Phys. 301 (2002) 31, hep-th/0205066.

[13] N. R. Constable, D. Z. Freedman, M. Headrick, S. Minwalla, L. Motl, A. Postnikov and W. Skiba, "PP-wave string interactions from perturbative Yang-Mills theory", JHEP 07 (2002) 017, hep-th/0205089.

[14] G. Arutyunov and E. Sokatchev, "Conformal fields in the pp-wave limit", JHEP 08 (2002) 014, hep-th/0205270.

[15] M. Bianchi, B. Eden, G. Rossi and Y. S. Stanev, "On operator mixing in $\mathcal{N}=4 S Y M "$ ", Nucl. Phys. B646 (2002) 69, hep-th/0205321.

[16] C.-S. Chu, V. V. Khoze and G. Travaglini, "Three-point functions in $\mathcal{N}=4$ Yang-Mills theory and pp-waves", JHEP 06 (2002) 011, hep-th/0206005

[17] A. Santambrogio and D. Zanon, "Exact anomalous dimensions of $\mathcal{N}=4$ Yang-Mills operators with large $R$ charge", Phys. Lett. B545 (2002) 425, hep-th/0206079

[18] M.-X. Huang, "String interactions in pp-wave from $\mathcal{N}=4$ Super Yang Mills", Phys. Rev. D66 (2002) 105002, hep-th/0206248.

[19] A. Parnachev and A. V. Ryzhov, "Strings in the near plane wave background and AdS/CFT", JHEP 10 (2002) 066, hep-th/0208010

[20] U. Gürsoy, "Vector operators in the BMN correspondence", hep-th/0208041.

[21] N. Beisert, C. Kristjansen, J. Plefka, G. W. Semenoff and M. Staudacher, "BMN correlators and operator mixing in $\mathcal{N}=4$ super Yang-Mills theory",

Nucl. Phys. B650 (2003) 125, hep-th/0208178

[22] N. R. Constable, D. Z. Freedman, M. Headrick and S. Minwalla, "Operator mixing and the BMN correspondence", JHEP 10 (2002) 068, hep-th/0209002.

[23] B. Eynard and C. Kristjansen, "BMN correlators by loop equations", JHEP 10 (2002) 027, hep-th/0209244.

[24] R. A. Janik, "BMN operators and string field theory", Phys. Lett. B549 (2002) 237, hep-th/0209263.

[25] T. Klose, "Conformal Dimensions of Two-Derivative BMN Operators", JHEP 03 (2003) 012, hep-th/0301150.

[26] H. Verlinde, "Bits, matrices and 1/N", hep-th/0206059.

[27] D. J. Gross, A. Mikhailov and R. Roiban, "A calculation of the plane wave string Hamiltonian from $\mathcal{N}=4$ super-Yang-Mills theory", hep-th/0208231.

[28] D. Vaman and H. Verlinde, "Bit strings from $\mathcal{N}=4$ gauge theory", hep-th/0209215.

[29] J. Pearson, M. Spradlin, D. Vaman, H. Verlinde and A. Volovich, "Tracing the String: $B M N$ correspondence at Finite $J^{2} / N$, hep-th/0210102 
[30] J. Gomis, S. Moriyama and J. Park, "SYM description of SFT Hamiltonian in a pp-wave background", hep-th/0210153

[31] S. Minwalla, "Restrictions imposed by superconformal invariance on quantum field theories", Adv. Theor. Math. Phys. 2 (1998) 781, hep-th/9712074

[32] G. Arutyunov and E. Sokatchev, "Implications of superconformal symmetry for interacting (2,0) tensor multiplets", Nucl. Phys. B635 (2002) 3, hep-th/0201145

[33] V. K. Dobrev and V. B. Petkova, "On the group theoretical approach to extended conformal supersymmetry: classification of multiplets", Lett. Math. Phys. 9 (1985) 287.

[34] L. Andrianopoli and S. Ferrara, "Short and long SU(2,2/4) multiplets in the AdS/CFT correspondence", Lett. Math. Phys. 48 (1999) 145, hep-th/9812067.

[35] M. Bianchi, S. Kovacs, G. Rossi and Y. S. Stanev, "Properties of the Konishi multiplet in $\mathcal{N}=4$ SYM theory", JHEP 05 (2001) 042, hep-th/0104016.

[36] S. J. Gates, Jr. and S. Vashakidze, "On $D=10, \mathcal{N}=1$ supersymmetry, superspace geometry and superstring effects", Nucl. Phys. B291 (1987) 172.

[37] M. F. Sohnius, "Bianchi identities for supersymmetric gauge theories", Nucl. Phys. B136 (1978) 461.

[38] S. J. Gates, Jr. and W. Siegel, "Understanding constraints in superspace formulations of supergravity", Nucl. Phys. B163 (1980) 519.

[39] S. J. Gates, Jr., K. S. Stelle and P. C. West, "Algebraic origins of superspace constraints in supergravity", Nucl. Phys. B169 (1980) 347.

[40] M. Bianchi, S. Kovacs, G. Rossi and Y. S. Stanev, "On the logarithmic behavior in $\mathcal{N}=4$ SYM theory", JHEP 08 (1999) 020, hep-th/9906188.

[41] G. Arutyunov, S. Frolov and A. C. Petkou, "Operator product expansion of the lowest weight CPOs in $\mathcal{N}=4$ SYM(4) at strong coupling", Nucl. Phys. B586 (2000) 547, hep-th/0005182.

[42] I. T. Todorov, M. C. Mintchev and V. B. Petkova, "Conformal invariance in quantum field theory", Sc. Norm. Sup. (1978), Pisa, Italy, 273p.

[43] J. Erdmenger and H. Osborn, "Conserved currents and the energy-momentum tensor in conformally invariant theories for general dimensions", Nucl. Phys. B483 (1997) 431, hep-th/9605009.

[44] H. Osborn, "N $=1$ superconformal symmetry in four-dimensional quantum field theory", Annals Phys. 272 (1999) 243, hep-th/9808041.

[45] J.-H. Park, "Superconformal symmetry and correlation functions", Nucl. Phys. B559 (1999) 455, hep-th/9903230

[46] P. S. Howe, E. Sokatchev and P. C. West, "3-point functions in $\mathcal{N}=4$ Yang-Mills", Phys. Lett. B444 (1998) 341, hep-th/9808162

[47] B. Eden, P. S. Howe and P. C. West, "Nilpotent invariants in $\mathcal{N}=4 S Y M "$, Phys. Lett. B463 (1999) 19, hep-th/9905085

[48] G. Arutyunov, S. Penati, A. C. Petkou, A. Santambrogio and E. Sokatchev, "Non-protected operators in $\mathcal{N}=4 S Y M$ and multiparticle states of AdS(5) SUGRA", Nucl. Phys. B643 (2002) 49, hep-th/0206020. 